\title{
RELATIVE CURRENTS
}

\author{
RADHIKA GUPTA
}

\begin{abstract}
In this paper we define currents relative to a free factor system. We prove that a fully irreducible outer automorphism relative to a free factor system acts with uniform north-south dynamics on a subspace of the space of projective relative currents.
\end{abstract}

\section{INTRODUCTION}

The study of the outer automorphism group $\operatorname{Out}(\mathbb{F})$ of a free group $\mathbb{F}$ of rank $n$ is highly motivated by the mapping class group $\operatorname{MCG}(\Sigma)$ of a surface $\Sigma$. The theory of $\operatorname{MCG}(\Sigma)$ has benefited greatly from its action on the curve complex $\mathcal{C}(\Sigma)$ which was proved to be hyperbolic in [MM99]. Analogously, Out(F) acts on the free factor complex $\mathcal{F F}_{n}$ which was proved to be hyperbolic in BF14. But sometimes the parallels between the two theories are not straightforward. For instance, consider a mapping class group element acting on $\mathcal{C}(\Sigma)$ with a fixed point, that is, it fixes a curve $\alpha$ on $\Sigma$. We can then look at its action on the curve complex of the subsurface given by the complement of $\alpha$ and understand it by an inductive process. On the other hand, consider an outer automorphism which fixes a free factor $A$ in $\mathcal{F F}_{n}$. Since the complement of $A$ in $\mathbb{F}$ is not well defined, we cannot pass to the free factor complex of a free group of lower rank. In [HM14], Handel and Mosher define a free factor complex relative to a free factor system (also called relative free factor complex) which is an Out(F)-analog of the curve complex of a subsurface. In the same paper, they also prove hyperbolicity of the relative free factor complex for a non-exceptional free factor system.

In this paper we develop the machinery of currents relative to a free factor system. This machinery is then used in Gup16 to classify the outer automorphisms that act with positive translation length on a relative free factor complex.

In Bon88, Bonahon first defined a space of geodesic currents for surfaces such that it contains the set of closed curves as a dense set. He studied the embedding of Teichmüller space in the space of geodesic currents and recovered Thurston's compactification of Teichmüller space. Currents for free groups were first studied by Reiner Martin Mar95] in his thesis. Analogous to geodesic currents, the space of currents for $\mathbb{F}$ contains the set of conjugacy classes of elements of $\mathbb{F}$ as a dense set. Currents for free groups have also been studied in Kap05, Kap06, KL09].

Let $\mathcal{A}$ be a non-trivial free factor system of $\mathbb{F}$. Here a non-trivial free factor system is one which is neither $\emptyset$ nor $\{[\mathbb{F}]\}$. In this paper we define a space of currents relative to $\mathcal{A}$ (also called relative currents) such that it contains the conjugacy classes of elements of $\mathbb{F}$ which are not contained in $\mathcal{A}$ as a dense set. Let $\partial^{2} \mathbb{F}$ be the

Received by the editors February 2, 2017, and, in revised form, July 8, 2017.

2010 Mathematics Subject Classification. Primary $20 \mathrm{~F} 65$.

The author was partially supported by the NSF grant of Mladen Bestvina (DMS-1607236). 
space of unoriented bi-infinite geodesics in a Cayley graph of $\mathbb{F}$. A relative current is a non-negative, additive, $\mathbb{F}$-invariant and flip-invariant function defined on the set of compact open sets of a subspace $\mathbf{Y}$ (Section 3.2) of $\partial^{2} \mathbb{F}$, which depends on $\mathcal{A}$. The subspace $\mathbf{Y}$ is defined in such a way that the action of $\mathbb{F}$ on $\mathbf{Y}$ is cocompact, which is important for the space of projectivized relative currents to be compact.

In order to show that the set of certain conjugacy classes of elements of $\mathbb{F}$ is dense in $\mathbb{P} \mathcal{R C}(\mathcal{A})$, we extend a relative current to a signed measured current which is in fact non-negative on words of bounded length (Section 8.1). We then follow the techniques of [Mar95].

Our main result is a generalization of a theorem in Mar95] (see also Uya14) which says that an atoroidal fully irreducible outer automorphism acts with uniform north-south dynamics on the space of projectivized currents, $\mathbb{P} \mathcal{M C}(\mathbb{F})$. In Mar95] it is also shown that a non-atoroidal fully irreducible outer automorphism acts with uniform north-south dynamics on a proper subspace of $\mathbb{P} \mathcal{M C}(\mathbb{F})$, which is given by the closure of primitive conjugacy classes of $\mathbb{F}$ in $\mathbb{P} \mathcal{M C}(\mathbb{F})$. In Uya15, Uyanik shows that a non-atoroidal fully irreducible acts with generalized north-south dynamics on $\mathbb{P} \mathcal{M C}(\mathbb{F})$. Generalizing Martin's results, we pass to a similarly defined subspace, denoted $\mathcal{M R C}(\mathcal{A})$, of $\mathbb{P} \mathcal{R C}(\mathcal{A})$.

Let $\operatorname{Out}(\mathbb{F}, \mathcal{A})$ be the subgroup of $\operatorname{Out}(\mathbb{F})$ containing outer automorphisms that preserve $\mathcal{A}$. After passing to a finite index subgroup of $\operatorname{Out}(\mathbb{F}, \mathcal{A})$ we can assume that each conjugacy class of free factor $[A]$ in $\mathcal{A}$ is invariant under elements of $\operatorname{Out}(\mathbb{F}, \mathcal{A})$. An outer automorphism $\Phi \in \operatorname{Out}(\mathbb{F}, \mathcal{A})$ is fully irreducible relative to $\mathcal{A}$ if no power of $\Phi$ fixes a non-trivial free factor system of $\mathbb{F}$ properly containing $\mathcal{A}$. Let $\zeta(\mathcal{A})$ be the sum of the number of conjugacy classes of free factors in $\mathcal{A}$ and the rank of a cofactor of $\mathcal{A}$.

Theorem A. Let $\mathcal{A}$ be a non-trivial free factor system of $\mathbb{F}$ with $\zeta(\mathcal{A}) \geq 3$. Let $\Phi \in \operatorname{Out}(\mathbb{F}, \mathcal{A})$ be fully irreducible relative to $\mathcal{A}$. Then $\Phi$ acts with uniform northsouth dynamics on $\mathcal{M R C}(\mathcal{A})$, that is, there are only two fixed points $\left[\eta_{\Phi}^{+}\right]$and $\left[\eta_{\Phi}^{-}\right]$and any compact set that does not contain $\left[\eta_{\Phi}^{-}\right]\left(\left[\eta_{\Phi}^{+}\right]\right)$uniformly converges to $\left[\eta_{\Phi}^{+}\right]\left(\left[\eta_{\Phi}^{-}\right]\right)$under $\Phi\left(\Phi^{-1}\right)$ iterates, respectively.

We use substitution dynamics techniques to understand the stable current $\eta_{\Phi}^{+}$ and the unstable current $\eta_{\Phi}^{-}$. In the absolute case (when $\mathcal{A}=\emptyset$ ), the transition matrix of a fully irreducible outer automorphism is primitive so Perron-Frobenius theory and the techniques in Que87, Chapter 5] can be used to define the stable and unstable current. Since the transition matrix of a relative fully irreducible outer automorphism is not primitive and the complement of $\mathcal{A}$ is not well-defined, some work needs to be done to define the limiting currents. Also some care is required to view an outer automorphism as a substitution due to presence of exceptional paths in a completely split train track representative. See Section 8.2 for details. We then study the legal and illegal turn structure of a conjugacy class under iteration by $\Phi$.

Our main application of Theorem $\mathrm{A}$ is the following result about loxodromic elements in the relative free factor complex $\mathcal{F} \mathcal{F}(\mathbb{F}, \mathcal{A})$.

Theorem B (Gup16). Let $\mathcal{A}$ be a non-exceptional free factor system of $\mathbb{F}$ and let $\Phi \in \operatorname{Out}(\mathbb{F}, \mathcal{A})$. Then $\Phi$ acts loxodromically on $\mathcal{F} \mathcal{F}(\mathbb{F}, \mathcal{A})$ if and only if $\Phi$ is fully irreducible relative to $\mathcal{A}$.

Plan of the paper. In Section 2, we review some basic definitions. In Section 3 , we define relative currents. In Section 4, we state the main proposition (from 
Section 8.2) about calculating frequencies of paths in a completely split train track representative of a relative fully irreducible outer automorphism. In Section 5, the stable and unstable relative currents are defined. In Section 6, we collect some lemmas about the legal and illegal turn structure of a conjugacy class under iterates by a relative fully irreducible outer automorphism. We conclude with the proof of the main theorem in Section 7. In the appendix we talk about substitution dynamics and extending relative currents to signed measured currents.

\section{Preliminaries}

2.1. Marked graphs and topological representatives. We review some terminology from BH92. Identify $\mathbb{F}$ with $\pi_{1}(\mathcal{R}, *)$, where $\mathcal{R}$ is a rose with $\mathfrak{n}$ petals and $\mathfrak{n}$ is the rank of $\mathbb{F}$. A marked graph $G$ is a graph of rank $\mathfrak{n}$, all of whose vertices have valence at least two, equipped with a homotopy equivalence $m: \mathcal{R} \rightarrow G$ called a marking. The marking determines an identification of $\mathbb{F}$ with $\pi_{1}(G, m(*))$.

A homotopy equivalence $\phi: G \rightarrow G$ induces an outer automorphism of $\pi_{1}(G)$ and hence an element $\Phi$ of $\operatorname{Out}(\mathbb{F})$. If $\phi$ sends vertices to vertices and the restriction of $\phi$ to edges is an immersion, then we say that $\phi$ is a topological representative of $\Phi$.

A filtration for a topological representative $\phi: G \rightarrow G$ is an increasing sequence of (not necessarily connected) $\phi$-invariant subgraphs $\emptyset=G_{0} \subset G_{1} \subset \cdots \subset G_{K}=G$. The closure of $G_{r} \backslash G_{r-1}$, denoted $H_{r}$ is a subgraph called the rth-stratum.

Let $\gamma$ be a reduced path in $G$. Then $\phi(\gamma)$ is the image of $\gamma$ under the map $\phi$. The tightened image of $\phi(\gamma)$ is denoted by $[\phi(\gamma)]$.

A path $\sigma$ is a periodic Nielsen path if the endpoints of $\sigma$ are fixed and $\phi^{k}(\sigma)$ is homotopic relative endpoints to $\sigma$, for some $k \geq 1$; the smallest such $k$ is called the period of $\sigma$. When $k=1$, then $\sigma$ is simply called a Nielsen path. A periodic Nielsen path is indivisible, denoted as INP, if it does not decompose as a concatenation of non-trivial Nielsen subpaths. A path $\sigma$ is a pre-Nielsen path if $\phi^{k}(\sigma)$ is a Nielsen path for some $k>0$.

2.2. Train track maps. We recall some more definitions from [BH92].

Let $G$ be a marked graph. A turn in $G$ is a pair of oriented edges of $G$ originating at a common vertex. A turn is non-degenerate if the edges are distinct, it is degenerate otherwise. A turn $\left(e_{1}, e_{2}\right)$ is contained in a filtration element $G_{r}$ if both $e_{1}$ and $e_{2}$ are contained in $G_{r}$. If $\gamma$ is an edge path given by $e_{1} e_{2} \ldots e_{m-1} e_{m}$, then we say that $\gamma$ contains the turn $\left(\overline{e_{i-1}}, e_{i}\right)$, where $\overline{e_{i}}$ denotes opposite orientation.

For $\phi: G \rightarrow G$ a topological representative and an edge $e$ in $G$, set $T \phi(e)$ equal to the first oriented edge of the edge path $\phi(e)$. Given a turn $\left(e_{1}, e_{2}\right)$, define $T \phi\left(e_{1}, e_{2}\right)=\left(T \phi\left(e_{1}\right), T \phi\left(e_{2}\right)\right)$. A turn is called illegal if under some iterate of $T \phi$ the turn maps to a degenerate turn, it is legal otherwise. A path $\gamma$ is called r-legal if all of its illegal turns are contained in $G_{r-1}$.

A matrix called transition matrix, denoted $M_{r}$, is associated to each stratum $H_{r}$. The $i j$ th entry of $M_{r}$ is the number of occurrences of the $i$ th edge of $H_{r}$ in either orientation in the image of the $j$ th edge under $\phi$. A non-negative matrix $M$ is called irreducible if for every $i, j$ there exists $k(i, j)>$ such that $i j$ th entry of $M^{k}$ is positive. A matrix is called primitive or aperiodic if there exists $k>0 \mathrm{such}$ that $M^{k}$ is positive. A stratum is called zero stratum if the transition matrix is the zero matrix. If $M_{r}$ is irreducible, then its Perron-Frobenius eigenvalue $\lambda_{r}$ is greater 
than or equal to 1 . Such a stratum is exponentially growing $(E G)$ if $\lambda_{r}>1$, it is called non-exponentially growing (NEG) otherwise.

A topological representative $\phi: G \rightarrow G$ of a free group outer automorphism $\Phi$ is a relative train track map with respect to a filtration $\emptyset=G_{0} \subset G_{1} \subset \cdots \subset G_{K}=G$ if $G$ has no valence-one vertices, if each non-zero stratum has an irreducible matrix and if each exponentially growing stratum satisfies the following conditions:

- If $E$ is an edge in $H_{r}$, then the first and the last edges in $\phi(E)$ are also in $H_{r}$.

- If $\gamma \in G_{r-1}$ is a non-trivial path with endpoints in $H_{r} \cap G_{r-1}$, then $[\phi(\gamma)]$ is non-trivial with endpoints in $H_{r} \cap G_{r-1}$.

- For each $r$-legal path $\beta \subset H_{r},[\phi(\beta)]$ is $r$-legal.

A reduced path $\sigma \subset G$ has height $r$ if the highest stratum it crosses is $G_{r}$.

2.3. Completely split train track maps (CT). In [FH11, Feighn and Handel defined completely split train track maps for outer automorphisms, which are better versions of relative train track maps. Instead of giving a complete definition, we list some facts which are used in this paper and then describe a complete splitting. Let $\phi: G \rightarrow G$ be a completely split train track map. The following facts proved in different papers can be found in HM13, Section 1.5.2].

\section{Facts 2.1.}

(1) Every periodic Nielsen path has period one.

(2) If $H_{r}$ is an EG stratum, then there is at most one indivisible Nielsen path (INP) in $G_{r}$ that intersects $H_{r}$ non-trivially.

(3) If $H_{r}$ is an EG stratum and if $\rho_{r}$ is an INP of height $r$, then $\rho_{r}$ crosses each edge of $H_{r}$ at least once, the initial oriented edges of $\rho_{r}$ and $\bar{\rho}_{r}$ are distinct oriented edges of $H_{r}$, and:

(a) $\rho_{r}$ is not closed if and only of it crosses some edge of $H_{r}$ exactly once and in this case:

(i) at least one endpoint of $\rho$ is not in $G_{r-1}$.

(ii) there does not exist a height $r$ fixed conjugacy class.

(b) $\rho_{r}$ is closed if and only if it crosses each edge of $H_{r}$ exactly twice, and in this case:

(i) the endpoint of $\rho_{r}$ is not in $G_{r-1}$.

(ii) the only height $r$ fixed conjugacy classes are those represented by $\rho_{r}$, its inverse and their iterates.

If $H_{r}$ is an EG stratum, then a non-trivial path in $G_{r-1}$ with endpoints in $H_{r} \cap G_{r-1}$ is called a connecting path. If an NEG stratum $H_{i}$ is a single edge $e_{i}$ such that $\phi\left(e_{i}\right)=e_{i} u_{i}$ for a non-trivial closed Nielsen path $u_{i}$, then $e_{i}$ is called a linear edge. Let $u_{i}=w_{i}^{d_{i}}$ for some $d_{i} \neq 0$ where $w_{i}$ is root-free. If $e_{i}$ and $e_{j}$ are distinct linear edges such that $\phi\left(e_{i}\right)=e_{i} w^{d_{i}}$ and $\phi\left(e_{j}\right)=e_{j} w^{d_{j}}$, where $d_{i} \neq d_{j}$ and $d_{i}, d_{j}>0$, then a path of the form $e_{i} w^{p} \overline{e_{j}}$, where $p \in \mathbb{Z}$, is called an exceptional path.

A decomposition of a path or a circuit $\sigma$ into subpaths is a called a splitting if one can tighten the image of $\sigma$ under $\phi$ by tightening the image of each subpath. In other words, there is no cancellation between images of two adjacent subpaths in the decomposition of $\sigma$.

Let $e$ be an edge in an irreducible stratum $H_{r}$ and let $k>0$. A maximal subpath $\sigma$ of $\left[\phi^{k}(e)\right]$ in a zero stratum $H_{i}$ is said to be $r$-taken. A non-trivial path or circuit 
in $G$ is said to be completely split if it has a splitting into subpaths each of which is either a single edge in an irreducible stratum, an indivisible Nielsen path, an exceptional path or a connecting path in a zero stratum $H_{i}$ that is taken and is maximal in $H_{i}$.

A relative train track map is completely split if for every edge $e$ in each irreducible stratum $\phi(e)$ is completely split and if $\sigma$ is a taken connecting path in a zero stratum, then $[\phi(\sigma)]$ is completely split.

2.4. Free factor system. A free factor system of $\mathbb{F}$ is a finite collection of proper free factors of $\mathbb{F}$ of the form $\mathcal{A}=\left\{\left[A_{1}\right], \ldots,\left[A_{k}\right]\right\}, k \geq 0$ such that there exists a free factorization $\mathbb{F}=A_{1} * \cdots * A_{k} * F_{N}$, where [.] denotes the conjugacy class of a subgroup. The free factor $F_{N}$ is referred to as the cofactor of $\mathcal{A}$, keeping in mind that it is not unique, even up to conjugacy. There is a partial ordering $\sqsubset$ on the set of free factor systems given as follows: $\mathcal{A} \sqsubset \mathcal{A}^{\prime}$ if for every $\left[A_{i}\right] \in \mathcal{A}$ there exists $\left[A_{j}^{\prime}\right] \in \mathcal{A}^{\prime}$ such that $A_{i} \subset A_{j}^{\prime}$ up to conjugation. The free factor systems $\emptyset$ and $\{[\mathbb{F}]\}$ are called trivial free factor systems. Define $\operatorname{rank}(\mathcal{A})$ to be the sum of the ranks of the conjugacy classes of free factors in $\mathcal{A}$ and let $\zeta(\mathcal{A})=k+N$.

The main geometric example of a free factor system is as follows: Suppose $G$ is a marked graph and $K$ is a subgraph whose non-contractible connected components are denoted $C_{1}, \ldots, C_{k}$. Let $\left[A_{i}\right]$ be the conjugacy class of a free factor of $\mathbb{F}$ determined by $\pi_{1}\left(C_{i}\right)$. Then $\mathcal{A}=\left\{\left[A_{1}\right], \ldots,\left[A_{k}\right]\right\}$ is a free factor system. We say $\mathcal{A}$ is realized by $K$ and denote it by $\mathcal{F}(K)$.

2.5. Relative free factor complex. Let $\mathcal{A}$ be a non-trivial free factor system of $\mathbb{F}$. In [HM14, the complex of free factor systems of $\mathbb{F}$ relative to $\mathcal{A}$, denoted $\mathcal{F} \mathcal{F}(\mathbb{F} ; \mathcal{A})$, is defined to be the geometric realization of the partial ordering $\sqsubset$ restricted to the set of non-trivial free factor systems $\mathcal{B}$ of $\mathbb{F}$ such that $\mathcal{A} \sqsubset \mathcal{B}$ and $\mathcal{A} \neq \mathcal{B}$. The exceptional free factor systems are certain ones for which $\mathcal{F} \mathcal{F}(\mathbb{F}, \mathcal{A})$ is either empty or zero-dimensional. They can be enumerated as follows:

- $\mathcal{A}=\left\{\left[A_{1}\right],\left[A_{2}\right]\right\}$ with $\mathbb{F}=A_{1} * A_{2}$. In this case $\mathcal{F} \mathcal{F}(\mathbb{F}, \mathcal{A})$ is empty.

- $\mathcal{A}=\{[A]\}$ with $\mathbb{F}=A * \mathbb{Z}$. In this case $\mathcal{F} \mathcal{F}(\mathbb{F}, \mathcal{A})$ is zero-dimensional.

- $\mathcal{A}=\left\{\left[A_{1}\right],\left[A_{2}\right],\left[A_{3}\right]\right\}$ with $\mathbb{F}=A_{1} * A_{2} * A_{3}$. In this case $\mathcal{F} \mathcal{F}(\mathbb{F}, \mathcal{A})$ is also zero-dimensional.

Theorem 2.2 ([HM14]). For any non-exceptional free factor system $\mathcal{A}$ of $\mathbb{F}$, the complex $\mathcal{F} \mathcal{F}(\mathbb{F}, \mathcal{A})$ is positive dimensional, connected and hyperbolic.

2.6. Fully irreducible relative to $\mathcal{A}$. Let $\mathcal{A}$ be a non-trivial free factor system. An outer automorphism $\Phi \in \operatorname{Out}(\mathbb{F}, \mathcal{A})$ is called irreducible relative to $\mathcal{A}$ if there is no non-trivial $\Phi$-invariant free factor system that properly contains $\mathcal{A}$. If every power of $\Phi$ is irreducible relative to $\mathcal{A}$, then we say that $\Phi$ is fully irreducible relative to $\mathcal{A}$ (or relative fully irreducible).

Let $\Phi \in \operatorname{Out}(\mathbb{F}, \mathcal{A})$. Then by Lemma 2.6.7 BFH00, there exists a relative train track map for $\Phi$, denoted $\phi: G \rightarrow G$, and filtration $\emptyset=G_{0} \subset G_{1} \subset \cdots \subset G_{r}=G$ such that $\mathcal{A}=\mathcal{F}\left(G_{s}\right)$ for some filtration element $G_{s}$. If $\Phi$ is fully irreducible relative to $\mathcal{A}$, then $\mathcal{A}=\mathcal{F}\left(G_{r-1}\right)$ and the top stratum $H_{r}$ is an EG stratum with Perron-Frobenius eigenvalue $\lambda_{\Phi}>1$. 


\subsection{Bounded cancellation constant and critical length.}

Lemma 2.3 ([Coo87]). Let $G$ be a marked metric graph and let $\phi: G \rightarrow G$ be a homotopy equivalence. There exists a constant $B C C(\phi)$, called the bounded cancellation constant, depending only on $\phi$ such that for any path $\rho$ in $G$ obtained by concatenating two paths $\alpha, \beta$, we have

$$
L(\phi(\rho)) \geq L(\phi(\alpha))+L(\phi(\beta))-B C C(\phi),
$$

where $L$ is the length function on $G$.

Let $B C C(\phi)$ be the bounded cancellation constant for $\phi: G \rightarrow G$, a relative train track representative of a relative fully irreducible outer automorphism $\Phi$ with top EG stratum $H_{r}$. The transition matrix of $H_{r}$ has a unique positive eigenvector whose smallest entry is one. For an edge $e_{i}$ in $H_{r}$, the eigenvector has an entry $v_{i}>0$. Assign a metric to $G$ such that each edge $e_{i}$ in $H_{r}$ is isometric to an interval of length $v_{i}$ and all edges in $G_{r-1}$ have length one. Then the $r$-length of edges in $H_{r}$ gets stretched by the PF eigenvalue $\lambda_{\Phi}$ under $\phi$. Let $l_{r}$ denote the $r$-length. Let $\alpha, \beta, \gamma$ be $r$-legal paths in $G$. Let $\alpha . \beta . \gamma$ be the path obtained by concatenating these $r$-legal paths. The only $r$-illegal turns possibly occur at the ends of the segments of $\beta$. Thus, if $\lambda_{\Phi} l_{r}(\beta)-2 B C C(\phi)>l_{r}(\beta)$, then iterations and tightening of $\alpha . \beta . \gamma$ will produce paths with $r$-length of legal segments corresponding to $\beta$ going to infinity. The constant $\frac{2 B C C(\phi)}{\lambda_{\Phi}-1}$ is called the critical r-length for $\phi$.

\section{Relative CURRENTS}

The goal of this section is to define the space of currents relative to a free factor system.

3.1. Boundary of $\mathbb{F}$. Given $\mathbb{F}$ and a fixed basis $\mathfrak{B}$ of $\mathbb{F}$, let $\operatorname{Cay}(\mathbb{F}, \mathfrak{B})$ be the Cayley graph of $\mathbb{F}$ with respect to $\mathfrak{B}$. The space of ends of the Cayley graph is called the boundary of $\mathbb{F}$, denoted by $\partial \mathbb{F}$. It is homeomorphic to the Cantor set. A one-sided cylinder set determined by a finite path $\gamma$ starting at the base point is the set of all rays starting at the base point that cross $\gamma$. Such cylinder sets form a basis for the topology on $\partial \mathbb{F}$ and are in fact both open and closed.

Let $\Delta$ denote the diagonal in $\partial \mathbb{F} \times \partial \mathbb{F}$. Let $\partial^{2} \mathbb{F}:=(\partial \mathbb{F} \times \partial \mathbb{F}-\Delta) / \mathbb{Z}_{2}$ be the space of unoriented bi-infinite geodesics in $\operatorname{Cay}(\mathbb{F}, \mathfrak{B})$. This space is also called the double boundary of $\mathbb{F}$. Finite paths $\gamma$ in $\operatorname{Cay}(\mathbb{F}, \mathfrak{B})$ determine two-sided cylinder sets, denoted $C(\gamma)$, which form a basis for the topology of $\partial^{2} \mathbb{F}$. Two-sided cylinder sets are open and compact and hence closed. Compact open sets are given by finite disjoint union of cylinder sets. Also $\partial^{2} \mathbb{F}$ is locally compact but not compact. The action of $\mathbb{F}$ on $\partial^{2} \mathbb{F}$ is cocompact.

Let $\mathcal{A}=\left\{\left[A_{1}\right], \ldots,\left[A_{k}\right]\right\}, k>0$, be a free factor system such that $\zeta(\mathcal{A}) \geq 3$.

Definition 3.1 (Relative basis). Choose representatives $A_{1}, \ldots, A_{k}$ of conjugacy classes of free factors in $\mathcal{A}$ such that $\mathbb{F}=A_{1} * \cdots * A_{k} * F_{N}$. Let $\mathfrak{B}_{\mathcal{A}}$ be a basis of $\mathbb{F}$ such that a basis of each $A_{i}$ is a subset of $\mathfrak{B}_{\mathcal{A}}$. Specifically,

$$
\mathfrak{B}_{\mathcal{A}}=\left\{a_{11}, \ldots a_{11_{s}}, \ldots, a_{i 1}, \ldots, a_{i i_{s}}, \ldots, a_{k 1}, \ldots, a_{k k_{s}}, b_{1}, \ldots, b_{p}\right\},
$$

where for a fixed $i$, the set $\left\{a_{i j}\right\}_{j=1}^{i_{s}}$ is a basis for $A_{i}$ and the set $\left\{b_{j}\right\}_{j=1}^{N}$ is a basis for $F_{N}$. Define a set $B_{\mathcal{A}}$ to be the collection of all words $a_{i j}^{ \pm} a_{k l}^{ \pm}$of length two such that $i \neq k$ and all the $b_{j}$ s. Note that if $\operatorname{rank}(\mathcal{A})=\operatorname{rank}(\mathbb{F})$, then the set of $b_{i} \mathrm{~s}$ is empty. We call $\mathfrak{B}_{\mathcal{A}}$ a relative basis of $\mathbb{F}$. 
Definition 3.2 (Double boundary of $\mathcal{A}$ ). Given a free factor $A$, define $\partial^{2} A$ to be the set of unoriented bi-infinite geodesics in $\partial^{2} \mathbb{F}$ in the closure of lifts of conjugacy classes of elements in $A$. Then define the double boundary of $\mathcal{A}$ as $\partial^{2} \mathcal{A}:=$ $\bigsqcup_{i=1}^{k} \partial^{2} A_{i}$.

Let $\mathbf{Y}=\partial^{2} \mathbb{F} \backslash \partial^{2} \mathcal{A}$. It inherits the subspace topology, denoted $\tau$, from $\partial^{2} \mathbb{F}$. It can also be given a topology, denoted $\tau^{\prime}$, where cylinder sets in $\mathbf{Y}$ determined by finite paths that cross at least one word in $B_{\mathcal{A}}$ form a basis for the topology. The two topologies are in fact equivalent. Indeed, for every $y \in \mathbf{Y}$ and every basis element $C(\gamma) \cap \mathbf{Y}$ of $\tau$ containing $y$ there is a basis element $C\left(\gamma^{\prime}\right)$ of $\tau^{\prime}$ containing $y$ such that $C\left(\gamma^{\prime}\right) \subset C(\gamma) \cap \mathbf{Y}$, where $\gamma$ and $\gamma^{\prime}$ are finite paths in Cay $\left(\mathbb{F}, \mathfrak{B}_{\mathcal{A}}\right), \gamma \subseteq \gamma^{\prime} \subset y$ and $\gamma^{\prime}$ crosses at least one word in $B_{\mathcal{A}}$. On the other hand, for every $y \in \mathbf{Y}$, and every basis element $C\left(\gamma^{\prime}\right)$ of $\tau^{\prime}$ containing $y$, the basis element $C\left(\gamma^{\prime}\right) \cap \mathbf{Y} \subset \partial^{2} \mathbb{F}$ of $\tau$ containing $y$ is such that $C\left(\gamma^{\prime}\right) \cap \mathbf{Y} \subseteq C\left(\gamma^{\prime}\right)$.

Lemma 3.3. $\mathbf{Y}$ is locally compact.

Proof. A space is locally compact if every point has a compact neighborhood. Let $x$ be an element of $\mathbf{Y}$. Take a finite subpath of $x$ that cannot be written as a string of words contained in a single free factor $A$, where $[A] \in \mathcal{A}$, and consider the cylinder set determined by that path. Then this cylinder set is a compact open set in $\mathbf{Y}$ containing $x$.

Lemma 3.4. The action of $\mathbb{F}$ on $\mathbf{Y}$ is cocompact.

Proof. Consider a compact set $C \subset \operatorname{Cay}\left(\mathbb{F}, \mathfrak{B}_{\mathcal{A}}\right)$ given by a finite union of cylinder sets determined by all paths with one endpoint at the origin and with label a word in $B_{\mathcal{A}}$. For every bi-infinite geodesic $\gamma$ in $\mathbf{Y}$ there is a $g \in \mathbb{F}$ such that $g \cdot \gamma$ crosses a path starting at the origin determined by a word in $B_{\mathcal{A}}$.

3.2. Definition of relative current. We first recall the definition of a measured current as defined in Mar95. A measured current is an additive, non-negative, $\mathbb{F}$-invariant and flip-invariant function on the set of compact open sets in $\partial^{2} \mathbb{F}$. It is uniquely determined by its values on the cylinder sets given by elements in $\mathbb{F}$.

Definition 3.5. With respect to the basis $\mathfrak{B}_{\mathcal{A}}$, let $\mathbb{F} \backslash \mathcal{A}$ denote the set of all words in $\mathbb{F}$ that are not contained in any free factor $A_{i}$, for $1 \leq i \leq k$. Note that $\mathbb{F} \backslash \mathcal{A}$ contains conjugates of words in $A_{i}$, as long as the conjugating elements are not in $A_{i}$.

Definition 3.6. Let $[\mathbb{F} \backslash \mathcal{A}]$ be the set of all conjugacy classes of elements in $\mathbb{F}$ that are not contained in any conjugacy class of a free factor in $\mathcal{A}$. Note that an element of $\mathbb{F} \backslash \mathcal{A}$ can be contained in the free product of distinct free factors representing elements of $\mathcal{A}$.

Let $\mathcal{C}(\mathbf{Y})$ be the collection of compact open sets in $\mathbf{Y}$. A relative current is an additive, non-negative, $\mathbb{F}$-invariant and flip-invariant function on $\mathcal{C}(\mathbf{Y})$. Let $\mathcal{R C}(\mathcal{A})$ denote the space of relative currents. A subbasis for the topology of $\mathcal{R C}(\mathcal{A})$ is given by the sets $\left\{\eta \in \mathcal{R C}(\mathcal{A}):\left|\eta(C)-\eta_{0}(C)\right| \leq \epsilon\right\}$, where $\eta_{0} \in \mathcal{R C}(\mathcal{A}), C \in \mathcal{C}(\mathbf{Y})$ and $\epsilon>0$.

Since $\partial^{2} \mathcal{A}$ is invariant under the action of $\operatorname{Out}(\mathbb{F}, \mathcal{A})$, the $\operatorname{action}$ of $\operatorname{Out}(\mathbb{F})$ on $\partial^{2} \mathbb{F}$ restricts to the action of $\operatorname{Out}(\mathbb{F}, \mathcal{A})$ on $\mathbf{Y}$. Thus, $\operatorname{Out}(\mathbb{F}, \mathcal{A})$ also acts on $\mathcal{C}(\mathbf{Y})$. 
The group $\operatorname{Out}(\mathbb{F}, \mathcal{A})$ acts on $\mathcal{R C}(\mathcal{A})$ as follows: Let $\eta \in \mathcal{R C}(\mathcal{A}), \Psi \in \operatorname{Out}(\mathbb{F}, \mathcal{A})$ and let $C \in \mathcal{C}(\mathbf{Y})$. Then

$$
\Psi . \eta(C)=\eta\left(\Psi^{-1}(C)\right) .
$$

A relative current can also be defined as an $\mathbb{F}$-invariant, locally finite, inner regular measure (called Radon measure) on the $\sigma$-algebra of Borel sets of $\mathbf{Y}$.

Lemma 3.7. A non-negative, additive function on $\mathcal{C}(\mathbf{Y})$ corresponds to a Radon measure on the Borel $\sigma$-algebra of $\mathbf{Y}$.

Proof. Given a non-negative, additive function $\eta$ on $\mathcal{C}(\mathbf{Y})$, define an outer measure $\eta^{*}: 2^{\mathbf{Y}} \rightarrow[0, \infty]$ as follows: For $A \in 2^{\mathbf{Y}}$

$$
\eta^{*}(A):=\inf \left\{\sum_{i=1}^{\infty} \eta\left(C_{i}\right): A \subseteq \bigcup_{i=1}^{\infty} C_{i} \text {, where } C_{i} \in \mathcal{C}(\mathbf{Y}) \text { is a cylinder set }\right\} .
$$

Using additivity of $\eta$ and compactness of $C$, we have $\eta^{*}(C)=\eta(C)$ for $C \in \mathcal{C}(\mathbf{Y})$. A cylinder set $C$ in $\mathcal{C}(\mathbf{Y})$ is outer measurable, that is, for every $A \in 2^{\mathbf{Y}}$ we have $\eta^{*}(A)=\eta^{*}\left(A \cap C^{c}\right)+\eta^{*}(A \cap C)$. An outer measure is a measure on the $\sigma$-algebra of outer measurable sets which in this case is the same as the $\sigma$-algebra of Borel sets. Therefore the outer measure $\eta^{*}$ is a measure on the Borel $\sigma$-algebra of $\mathbf{Y}$. The space $\mathbf{Y}$ is locally compact and Hausdorff and every open set in $\mathbf{Y}$ is $\sigma$-compact, that is, a countable union of compact sets. Also $\eta^{*}$ is a non-negative Borel measure on $\mathbf{Y}$ such that it is finite on compact sets. Therefore, $\eta^{*}$ is a regular measure.

Thus the space of relative currents can be given a weak-* topology, that is, $\eta_{n} \rightarrow \eta$ in $\mathcal{R C}(\mathcal{A})$ iff $\int_{\mathbf{Y}} f d \eta_{n} \rightarrow \int_{\mathbf{Y}} f d \eta$ for all compactly supported functions $f$ on $\mathbf{Y}$. Since $\mathbf{Y}$ is a locally compact space, by the result in Bou65, Chapter III, Section 1] $\mathcal{R C}(\mathcal{A})$ is complete.

3.3. Coordinates for $\mathcal{R C}(\mathcal{A})$. Fix a relative basis $\mathfrak{B}_{\mathcal{A}}$ of $\mathbb{F}$. Given $w \neq 1 \in \mathbb{F}$, consider the unique oriented path, denoted $\gamma_{w}$, determined by $w$ starting at the base point and let $C(w)$ denote $C\left(\gamma_{w}\right)$. Note that this cylinder set contains unoriented bi-infinite geodesics that cross $\gamma_{w}$. For $w \in \mathbb{F} \backslash \mathcal{A}$, we have $C(w) \subset \mathcal{C}(\mathbf{Y})$. Orbits of cylinder sets of the form $C(w)$ under deck transformations cover $\mathbf{Y}$. We denote $\eta$ applied to $C(w)$ by $\eta(w)$.

- Let $v \in \mathbb{F}$. Then $v \cdot C(w)$ is the set of all bi-infinite geodesics that cross an edge path labeled by $w$ starting at the vertex labeled $v$ in the Cayley graph. By $\mathbb{F}$-invariance of a relative current we have that $\eta(C(w))=\eta(v \cdot C(w))$. Thus we can work just with the cylinder sets determined by finite paths starting at the base point. Since every compact open set is a finite disjoint union of cylinder sets, a relative current is uniquely determined by its values on (cylinder sets determined by) words in $\mathbb{F} \backslash \mathcal{A}$.

- Since a relative current is uniquely determined by its values on $\mathbb{F} \backslash \mathcal{A}$, a sequence of relative currents $\eta_{n}$ converges to $\eta$ if and only if $\eta_{n}(w) \rightarrow \eta(w)$ for all $w \in \mathbb{F} \backslash \mathcal{A}$.

- For any finite path $\gamma$ in $\operatorname{Cay}\left(\mathbb{F}, \mathfrak{B}_{\mathcal{A}}\right)$ we have $C(\gamma)=C(\bar{\gamma})$, where $\bar{\gamma}$ denotes the opposite orientation on $\gamma$. If $w$ and $\gamma_{w}$ are as above, then $C(w)=$ $C\left(\gamma_{w}\right)=C\left(\overline{\gamma_{w}}\right)=w \cdot C(\bar{w})$. Thus $\eta(w)=\eta(\bar{w})$.

- Let $w=e_{0} e_{1} \ldots e_{l} \in \mathbb{F} \backslash \mathcal{A}$, where each $e_{i} \in \mathfrak{B}_{\mathcal{A}}^{ \pm}$. Then $C(w)=\bigcup C(w e)$, where the union is taken over all basis elements in $\mathfrak{B}_{\mathcal{A}}$ except $e=\overline{e_{l}}$. Here $\bar{e}$ denotes the inverse of $e$. Also $C(w)=\bigcup \bar{e} \cdot C(e w)$, where $e$ is any basis 
element other than $\overline{e_{0}}$. Then additivity of a relative current can be stated as

$$
\eta(w)=\sum_{e \neq \overline{e_{l}}} \eta(w e) \quad \text { or } \quad \eta(w)=\sum_{e \neq \overline{e_{0}}} \eta(e w) .
$$

For example, let $\mathbb{F}=\langle a, b\rangle$ and $\mathcal{A}=\{[\langle a\rangle]\}$, we have

$$
\begin{aligned}
& \eta(b)=\eta(b a)+\eta(b b)+\eta(b \bar{a}), \\
& \eta(b)=\eta(a b)+\eta(b b)+\eta(\bar{a} b) .
\end{aligned}
$$

- Let $v, w \in \mathbb{F} \backslash \mathcal{A}$ be such that $v$ is a subword of $w$. Then $\eta(w) \leq \eta(v)$.

Example 3.8 (Relative current). Consider a conjugacy class $\alpha \in[\mathbb{F} \backslash \mathcal{A}]$ such that $\alpha$ is not a power of any other conjugacy class in $\mathbb{F}$. Then $\eta_{\alpha}(w)$ is the number of occurrences of $w$ in the cyclic words $\alpha$ and $\bar{\alpha}$. Equivalently, one can also count the number of lifts of $\alpha$ that cross the path $\gamma_{w}$ in the Cayley graph. We call such currents and their multiples rational relative currents. For example, let $\mathbb{F}=$ $\langle a, b\rangle, \mathcal{A}=\{[\langle a\rangle]\}$ and let $\alpha=a b a \bar{b} a b$. Then $\eta_{\alpha}(b)=3, \eta_{\alpha}(b a)=2, \eta_{\alpha}(a b a b)=1$ and $\eta_{\alpha}(\bar{b} a b)=1$.

Given $w \in \mathbb{F}$, a length $k$ extension of $w$ is a word $w^{\prime}=w x_{1} \ldots x_{k}$, where $x_{i} \in \mathfrak{B}_{\mathcal{A}}$, $x_{i} \neq \overline{x_{i+1}}$ and $x_{1}$ is not the inverse of the last letter of $w$.

Lemma 3.9. Any non-negative function $\eta$ on $\mathbb{F} \backslash \mathcal{A}$ invariant under inversion and the action of $\mathbb{F}$, and satisfying the condition

$$
\eta(w)=\sum_{\begin{array}{c}
\text { length one } \\
\text { extension of } w
\end{array}} \eta(v)
$$

for all $w \in \mathbb{F} \backslash \mathcal{A}$ determines a relative current.

Proof. A set $C \in \mathcal{C}(\mathbf{Y})$ can be written as a disjoint union of cylinder sets $C\left(w_{1}\right), \ldots$, $C\left(w_{k}\right)$ with $w_{i} \in \mathbb{F} \backslash \mathcal{A}$. Then define $\eta(C):=\sum_{i=1}^{k} \eta\left(w_{i}\right)$. The value $\eta(C)$ does not depend on the choice of $w_{i}$. Thus we have an additive and non-negative function on $\mathcal{C}(\mathbf{Y})$ which is invariant under the action of $\mathbb{F}$.

3.4. Projectivized relative currents. Let $\mathbb{P} \mathcal{R C}(\mathcal{A})$ denote the space of projectivized relative currents. It has quotient topology induced from $\mathcal{R C}(\mathcal{A})$. A sequence of projective currents $\left[\eta_{i}\right]$ converges to $[\eta]$ in $\mathbb{P} \mathcal{R C}(\mathcal{A})$ iff there exist scaling constants $a_{i}$ such that relative currents $a_{i} \eta_{i}$ converge to $\eta$ in $\mathcal{R C}(\mathcal{A})$.

Example 3.10. Let $\mathbb{F}=\langle a, b\rangle$ and let $\mathcal{A}=\{[\langle a\rangle]\}$. Consider the sequence $\eta_{a^{n} b} \in \mathcal{R C}(\mathcal{A})$. This sequence converges to a relative current $\eta_{\infty}$ which is given by $\eta_{\infty}\left(a^{m} b a^{n}\right)=1$ for all $m, n \geq 0$ and $\eta_{\infty}(w)=0$ for all other $w \in \mathbb{F} \backslash \mathcal{A}$. Whereas in the space of measured currents as defined in Mar95, the sequence $\eta_{a^{n} b} / n$ converges to the current $\eta_{a}$.

Lemma 3.11. $\mathbb{P} \mathcal{R C}(\mathcal{A})$ is compact.

Proof. Consider a sequence of projective relative currents $\left[\eta_{n}\right]$. We have to show that it has a convergent subsequence. Fix a relative basis $\mathfrak{B}_{\mathcal{A}}$ and the associated set $B_{\mathcal{A}}=\left\{u_{1}, \ldots, u_{r}\right\}$ (see Definition [3.1). Let $\eta_{n}$ be a representative of $\left[\eta_{n}\right]$ normalized such that $\eta_{n}\left(u_{i}\right) \leq 1$ for all $u_{i} \in B_{\mathcal{A}}$ and $\eta_{n}\left(u_{j}\right)=1$ for some $u_{j} \in B_{\mathcal{A}}$. We have $\eta_{n}(w) \leq \eta_{n}\left(u_{i}\right)$ where $w \in \mathbb{F} \backslash \mathcal{A}$ and crosses a path labeled $u_{i} \in B_{\mathcal{A}}$ in $\operatorname{Cay}\left(\mathbb{F}, \mathfrak{B}_{\mathcal{A}}\right)$. The bounded sequence $\left\{\left(\eta_{n}\left(u_{1}\right), \ldots, \eta_{n}\left(u_{r}\right)\right)\right\}_{n \in \mathbb{N}}$ has a subsequence 
that converges to a non-zero element of $\mathbb{R}^{r}$. For every $w \in \mathbb{F} \backslash \mathcal{A},\left\{\eta_{n}(w)\right\}_{n \in \mathbb{N}}$ is a bounded sequence and hence has a convergent subsequence. Now using the diagonal argument conclude that $\left\{\left(\eta_{n}(w)\right)_{w \in \mathbb{F} \backslash \mathcal{A}}\right\}_{n \in \mathbb{N}}$ has a subsequence that converges to a non-zero element. Thus $\left\{\left[\eta_{n}\right]\right\}_{n \in \mathbb{N}}$ has a convergent subsequence in $\mathbb{P} \mathcal{R C}(\mathcal{A})$.

\subsection{Density of rational relative currents.}

Proposition 3.12. The set of projectivized relative currents induced by conjugacy classes $\alpha \in[\mathbb{F} \backslash \mathcal{A}]$ are dense in $\mathbb{P} \mathcal{R C}(\mathcal{A})$.

Let $\mathfrak{B}_{\mathcal{A}}$ be a relative basis of $\mathbb{F}$ and let $|w|$ denote the word length of $w \in \mathbb{F}$ with respect to $\mathfrak{B}_{\mathcal{A}}$. In the absolute case, the following lemma is the main step to prove density of rational currents. But it doesn't directly apply to the relative setting as explained below.

Lemma 3.13 ([Mar95, Lemma 15]). Let $\eta$ be a measured current and let $k \geq 2$. Let $P=2 \mathfrak{n}(2 \mathfrak{n}-1)^{2 \mathfrak{n}(2 \mathfrak{n}-1)^{k-2}}$ be a constant. If $\eta\left(w_{0}\right) \geq P$ for some $w_{0} \in \mathbb{F}$ with $\left|w_{0}\right|=k$, then there exists a conjugacy class $\alpha \in[\mathbb{F}]$ and the corresponding measured current $\eta_{\alpha}$ with $\eta(w) \geq \eta_{\alpha}(w)$ for all $w \in \mathbb{F}$ and $|w| \leq k$.

The proof of the above lemma relies on finding cycles in a certain labeled directed graph associated to $\eta$ defined as follows: vertices are given by words of length $k-1$ and edges are given by words of length $k$. A directed edge $w$ joins vertex $u$ to vertex $v$ if $u$ is the prefix of $w$ and $v$ is the suffix of $w$. An edge $w$ is labeled by $\eta(w)$. Since $\eta$ satisfies additivity laws for all words in $\mathbb{F}$, this graph satisfies Kirchhoff's law at each vertex which is crucial to find cycles (which correspond to $\alpha$ ) in the graph. The same graph defined for a relative current $\eta_{0}$ does not satisfy Kirchhoff's law at vertices which correspond to words in some free factor $A_{i}$ for $\left[A_{i}\right] \in \mathcal{A}$ because $\eta_{0}$ is not defined for words in $\mathcal{A}$.

A signed measured current on $\partial^{2} \mathbb{F}$ is an $\mathbb{F}$-invariant and additive function on the set of compact open sets of $\partial^{2} \mathbb{F}$. We now restate the above lemma for a signed measured current which is non-negative on words in $\mathbb{F}$ of bounded length.

Lemma 3.14. Let $k \geq 2$ and let $\eta$ be a signed measured current such that $\eta(w) \geq 0$ for all $w \in \mathbb{F}$ with $|w| \leq k$. Let $P=2 \mathfrak{n}(2 \mathfrak{n}-1)^{2 \mathfrak{n}(2 \mathfrak{n}-1)^{k-2}}$ be a constant. If $\eta\left(w_{0}\right) \geq P$ for some $w_{0} \in \mathbb{F}$ with $\left|w_{0}\right|=k$, then there exists a conjugacy class $\alpha \in[\mathbb{F}]$ and the corresponding measured current $\eta_{\alpha}$ with $\eta(w) \geq \eta_{\alpha}(w)$ for all $w \in \mathbb{F}$ and $|w| \leq k$.

For $\eta_{0} \in \mathcal{R C}(\mathcal{A})$, let $\eta$ be a signed measured current such that $\eta(w)=\eta_{0}(w)$ for $w \in \mathbb{F} \backslash \mathcal{A}$ and $\eta(w) \geq 0$ for all words $w \in \mathbb{F}$ with $|w| \leq k$. We call such an $\eta$ a $k$-extension of $\eta_{0}$.

Lemma 3.15. Let $\eta_{0}$ be a relative current and let $k \geq 1$. Then there exists a signed measured current $\eta$ which is a $k$-extension of $\eta_{0}$.

To prove the above lemma, we start by defining $\eta$ on length one words in $\mathcal{A}$ arbitrarily and then extending the current to length two words by imposing the additivity property. It needs to be checked that the constraints obtained from the additive property are consistent. A detailed proof is given in Section 8.1 Assuming the above lemma is true we now prove Proposition 3.12 .

Proof of Proposition 3.12. We follow the same method of proof as in [Mar95. Let $\eta_{0}$ be a relative current and let $k \geq 2$. Choose $R>0$ such that $R \eta_{0}\left(w_{0}\right) \geq P$ for 
some $w_{0} \in \mathbb{F} \backslash \mathcal{A}$ with $\left|w_{0}\right|=k$. Consider a signed measured current $\eta$ which is a $k$-extension of $\eta_{0}$. Then by Lemma 3.14 applied to $R \eta$, there exists an $\alpha_{1} \in \mathbb{F}$ such that $R \eta(w) \geq \eta_{\alpha_{1}}(w)$ for all $w \in \mathbb{F}$ with $|w| \leq k$. If $R \eta(w) \leq \eta_{\alpha_{1}}(w)+P$ for all $w \in \mathbb{F}$ with $|w| \leq k$, then we stop, otherwise we again apply Lemma 3.14 to $R \eta-\eta_{\alpha_{1}}$ to obtain $\alpha_{2} \in \mathbb{F}$ such that $R \eta(w)-\eta_{\alpha_{1}}(w) \geq \eta_{\alpha_{2}}(w)$ for all $w \in \mathbb{F}$ with $|w| \leq k$. By induction, we have $\sum \eta_{\alpha_{i}}(w) \leq R \eta(w) \leq \sum \eta_{\alpha_{i}}(w)+P$ for all words of length less than or equal to $k$.

It is necessary that at least one of the $\alpha_{i} \in[\mathbb{F} \backslash \mathcal{A}]$. Indeed, if they were all in $\mathcal{A}$, then $\sum \eta_{\alpha_{i}}\left(w_{0}\right)=0$ which would mean $R \eta\left(w_{0}\right) \leq P$, which is a contradiction.

Now we have that

$$
\left|\eta(w)-\frac{\sum \eta_{\alpha_{i}}(w)}{R}\right| \leq \frac{P}{R}
$$

for all $w \in \mathbb{F}$ with $|w| \leq k$. For $w \in \mathbb{F} \backslash \mathcal{A}$, in fact,

$$
\left|\eta_{0}(w)-\frac{\sum_{\alpha_{i} \notin \mathcal{A}} \bar{\eta}_{\alpha_{i}}(w)}{R}\right| \leq \frac{P}{R}
$$

where $\bar{\eta}_{\alpha_{i}}$ is the restriction of $\eta_{\alpha_{i}}$ to $\mathbf{Y}$.

Since $R$ can be chosen arbitrarily large we can approximate relative currents by sums of rational relative currents for all $w \in \mathbb{F} \backslash \mathcal{A}$ with $|w| \leq k$. Now we can approximate $\sum_{\alpha_{i} \notin \mathcal{A}} \eta_{\alpha_{i}}$ by $\frac{1}{m} \eta_{\beta^{m}}$, where $\beta^{m}=w_{1}^{m} w_{2}^{m} \cdots w_{l}^{m}$ and $w_{i}$ is in the conjugacy class of $\alpha_{i}$.

\subsection{Relative Whitehead graph.}

Definition 3.16. A conjugacy class $\alpha \in[\mathbb{F} \backslash \mathcal{A}]$ is $\mathcal{A}$-separable if it is contained in a non-trivial free factor system containing $\mathcal{A}$. Topologically, $\alpha$ is $\mathcal{A}$-separable if there is an $\mathbb{F}$-tree $T$ with set of vertex stabilizers given by $\mathcal{A}$ such that an axis of $\alpha$ does not cross every orbit of edges.

To detect when a conjugacy class is $\mathcal{A}$-separable, we use Whitehead's algorithm and a theorem of Stallings [Sta99]. As defined in Sta99, a collection $\mathcal{C}$ of conjugacy classes in $[\mathbb{F}]$ is separable if there exist free factors $F, F^{\prime}$ such that $\mathbb{F}=F * F^{\prime}$ and each conjugacy class in $\mathcal{C}$ is contained in either $F$ or $F^{\prime}$. Let $\alpha_{i} \in\left[A_{i}\right], 0<i \leq k$, be a conjugacy class such that $\alpha_{i}$ is not contained in any proper free factor of $\left[A_{i}\right]$. We say $\alpha_{i}$ is filling in $\left[A_{i}\right]$.

Lemma 3.17. A conjugacy class $\alpha \in[\mathbb{F} \backslash \mathcal{A}]$ is $\mathcal{A}$-separable if and only if the collection of conjugacy classes $\mathcal{C}=\left\{\alpha, \alpha_{1}, \ldots, \alpha_{k}\right\}$ is separable.

Proof. If $\mathcal{C}$ is separable, then there exist a decomposition $\mathbb{F}=F * F^{\prime}$ such that each conjugacy class in $\mathcal{C}$ is contained either in $F$ or $F^{\prime}$. Suppose $\alpha_{i} \in F$. Then we claim that $A_{i}$ is contained in $F$ up to conjugation. Suppose not. We have that $F \cap A_{i} \neq \emptyset$ up to conjugation. Also the intersection of two free factors is a free factor. So $\alpha_{i}$ is contained in a non-trivial free factor of $A_{i}$, which is a contradiction. Thus $\left\{[F],\left[F^{\prime}\right]\right\}$ is a non-trivial free factor system containing $\mathcal{A}$ that contains the conjugacy class $\alpha$.

On the other hand, if $\alpha$ is contained in a proper free factor system $\mathcal{D}$ containing $\mathcal{A}$, then $\mathcal{C}$ is separable.

Definition 3.18 (Whitehead graph [Whi36]). Given a basis $\mathfrak{B}$ of $\mathbb{F}$, the Whitehead graph of a collection $\mathcal{C}$ of conjugacy classes, denoted $W h(\mathcal{C})$, is defined as 
follows: the vertices are given by basis elements and their inverses. There is an edge connecting vertices $x$ and $y$ if $\bar{x} y$ is a subword of a conjugacy class in $\mathcal{C}$.

Theorem 3.19 ([Sta99, Theorem 4.2]). Let $\mathcal{C}$ be a collection of conjugacy classes in $[\mathbb{F}]$. If $W h(\mathcal{C})$ is connected and $\mathcal{C}$ is separable, then there is a cut vertex in $W h(\mathcal{C})$.

Definition 3.20 (Relative Whitehead graph). For each $\left[A_{i}\right] \in \mathcal{A}$ fix filling conjugacy classes $\alpha_{i} \in\left[A_{i}\right]$. The relative Whitehead graph of a conjugacy class $\alpha \in[\mathbb{F} \backslash \mathcal{A}]$, denoted $W h(\alpha, \mathcal{A})$, is defined as the Whitehead graph of the collection $\left\{\alpha, \alpha_{1}, \ldots, \alpha_{k}\right\}$.

Note that even though we fix some filling conjugacy classes to define the relative Whitehead graph, detecting $\mathcal{A}$-separability of $\alpha$ is independent of them by Lemma 3.17

3.7. A closed subspace of $\mathbb{P} \mathcal{R C}(\mathcal{A})$. In the absolute case, when a fully irreducible outer automorphism $\Psi$ is a pseudo-Anosov on a surface with one boundary component, the measured current corresponding to the boundary conjugacy class in the space of projectivized measured currents $\mathcal{M C}(\mathbb{F})$ is fixed under the action of $\Psi$. Thus in Mar95, a closed subspace is considered which is the closure of all primitive conjugacy classes in $\mathcal{M C}(\mathbb{F})$. For the same reason, we pass to a smaller closed $\operatorname{Out}(\mathbb{F}, \mathcal{A})$-invariant subspace of $\mathbb{P} \mathcal{R C}(\mathcal{A})$. Let

$$
\mathcal{M R C}(\mathcal{A})=\overline{\left\{\left[\eta_{\alpha}\right] \in \mathbb{P R C}(\mathcal{A}) \mid \alpha \text { is } \mathcal{A} \text {-separable }\right\}} .
$$

Lemma 3.21. $\left[\eta_{\alpha}\right] \in \mathbb{P} \mathcal{R C}(\mathcal{A})$ is in $\mathcal{M R C}(\mathcal{A})$ if and only if $\alpha$ is $\mathcal{A}$-separable.

Proof. Let us assume that $\alpha$ is not $\mathcal{A}$-separable. Then by Theorem 3.19 , the relative Whitehead graph of $\alpha$ with respect to any relative basis is connected without a cut vertex. Let $w_{\alpha} \in \mathbb{F} \backslash \mathcal{A}$ be a cyclically reduced representative of $\alpha$. Consider a relative current $\eta_{v}$ where $v \in[\mathbb{F} \backslash \mathcal{A}]$ such that $\eta_{v}\left(w_{\alpha}^{2}\right)>0$. This means that any relative Whitehead graph of $v$ contains the Whitehead graph of $\alpha$ as a subgraph and hence is connected without cut vertices. By Theorem 3.19 and Lemma 3.17, this implies that $v$ is not $\mathcal{A}$-separable. Thus $\eta_{v}\left(w_{\alpha}^{2}\right)=0$ for all $\mathcal{A}$-separable conjugacy classes $v$ in $[\mathbb{F} \backslash \mathcal{A}]$, which in turn implies that $\eta\left(w_{\alpha}\right)=0$ for any $[\eta] \in \mathcal{M R C}(\mathcal{A})$. Since $\eta_{\alpha}\left(w_{\alpha}^{2}\right)>0$, we have that $\eta_{\alpha} \notin \mathcal{M R C}(\mathcal{A})$.

\section{Substitution Dynamics}

In Que87, a theory of substitution dynamics is developed for primitive substitutions to study their limiting behavior. This theory can be used to study a fully irreducible outer automorphism by viewing it as a substitution. In Section 8.2 of the Appendix, we develop a theory of substitution dynamics for a different class of substitutions in order to study outer automorphisms relative to a free factor system. Here we state the main result (Proposition 8.13) from Section 8.2

For $\gamma$ and $\alpha$ two paths in a graph $G$, let $(\gamma, \alpha)$ be the number of occurrences of $\gamma$ in $\alpha$.

Proposition 4.1. Let $\phi: G \rightarrow G$ be a completely split train track map. Let a be an edge in an EG stratum $H_{r}$ such that $\phi(a)$ starts with $a$, and let $\rho_{a}:=\lim _{n \rightarrow \infty} \phi^{n}(a)$. Let $\gamma$ be a path in $G_{r}$ that crosses $H_{r}$. Then

$$
\lim _{n \rightarrow \infty} \frac{\left(\gamma, \phi^{n}(a)\right)}{\lambda^{n}}=: d_{\gamma, a}
$$


exists and is non-negative. Here $\lambda$ is the Perron-Frobenius eigenvalue of the aperiodic $E G$ stratum $H_{r}$. If $b \in H_{r}$ is another edge, then for every $\gamma$ as above,

$$
d_{\gamma, b}=\kappa d_{\gamma, a},
$$

where $\kappa$ is a constant with $\kappa=\kappa\left(a, b,\left.\phi\right|_{H_{r}}\right)$.

In general, it is possible that $\gamma$ grows faster than $\lambda$ due to the presence of subpaths in $G_{r-1}$ that grow faster. The point of the above proposition is to ignore the contribution to the growth of $\gamma$ from the lower stratum but still be able to compute frequencies of paths that cross $H_{r}$ and are not necessarily completely contained in $H_{r}$.

\section{Stable and unstable Relative Current}

In this section, we define the stable and unstable relative currents associated to a fully irreducible outer automorphism relative to $\mathcal{A}$. Before we state the general result let us look at some examples. The three examples that follow illustrate the cases when the growth in the stratum corresponding to $\mathcal{A}$ is less than, greater than and equal to the growth in the top EG stratum.

Example 5.1. Let $F_{3}=\langle a, b, c\rangle$. Let $G$ be the rose on three petals labeled $a, b$ and $c$. Consider an outer automorphism $\Phi$ given by a train track representative $\phi: G \rightarrow G$, where

$$
\phi(a)=a, \phi(b)=b a c, \phi(c)=c b a c .
$$

Let $\mathcal{A}=\{[\langle a\rangle]\}$. The transition matrix for $\phi$ is given by

$$
M=\left[\begin{array}{ccc}
b & c & a \\
1 & 1 & 0 \\
1 & 2 & 0 \\
1 & 1 & 1
\end{array}\right]
$$

Note that $\Phi$ is not fully irreducible relative to $\mathcal{A}$ because the free factor system $\{[\langle b, a c\rangle],[\langle a\rangle]\}$ is $\Phi$-invariant. But it is still instructive to understand the limiting behavior in this simple case.

Let $\rho_{b}=\lim _{n \rightarrow \infty} \phi^{n}(b)$ be a ray that is fixed by $\phi$. We can view $\phi$ as a substitution $\zeta$ on the alphabet $\mathbb{A}=\{a, b, c\}$. Let $\mathbb{A}_{l}$ be the set of words of length $l$ on $\mathbb{A}$ that appear in $\rho_{b}$. For example, $\mathbb{A}_{2}=\{b a, c a, c b, a c\}$. Note that the sets $\mathbb{A}_{l}$ are independent of the specific choice $b$. We define a substitution $\zeta_{l}$ on $\mathbb{A}_{l}$ as follows: let $w \in \mathbb{A}_{l}$ start with $x \in \mathbb{A}$. Then $\zeta_{l}(w)$ consists of the ordered list of the first $|\zeta(x)|$ subwords of length $l$ of the word $\zeta(w)$. For example, $\zeta_{2}(b a)=b a \cdot a c \cdot c a$. Let $M_{l}$ be the transition matrix of $\zeta_{l}$ and let $\mathcal{B}_{l}$ be the transition matrix for $\zeta_{l}$ restricted to words in $\mathbb{F} \backslash \mathcal{A}$. We want to calculate the frequency of occurrences of words in $\mathbb{F} \backslash \mathcal{A}$ that appear in $\rho_{b}$.

Let $w \in \mathbb{A}_{l}$ and let $\beta$ be a word of length $l$ that starts with $b$. Then

$$
\lim _{n \rightarrow \infty} \frac{\left(w, \phi^{n}(b)\right)}{\lambda^{n}}=\lim _{n \rightarrow \infty} \frac{M_{l}^{n}(w, \beta)}{\lambda^{n}}=\lim _{n \rightarrow \infty} \frac{\mathcal{B}_{l}^{n}(w, \beta)}{\lambda^{n}}=: d_{w, b} .
$$


Here $\lambda$ is the PF-eigenvalue of the top EG stratum. See Section 8.2 for a detailed explanation. For example, in length one and two we have

$$
\begin{aligned}
& \text { ba } c a c b \text { ac } \\
& \mathcal{B}_{1}=\left[\begin{array}{cc}
b & c \\
1 & 1 \\
1 & 2
\end{array}\right], \quad \mathcal{B}_{2}=\left[\begin{array}{llll}
1 & 1 & 1 & 0 \\
1 & 1 & 0 & 0 \\
0 & 1 & 2 & 0 \\
1 & 1 & 1 & 1
\end{array}\right]
\end{aligned}
$$

We take $\beta=b$ and $\beta=b a$ for length one and length two words, respectively. Then

$$
\begin{array}{r}
\left(b, \rho_{b}\right)=\frac{(5-\sqrt{5})}{10}, \quad\left(c, \rho_{b}\right)=\frac{1}{\sqrt{5}}, \\
\left(a c, \rho_{b}\right)=\frac{1}{\sqrt{5}}, \quad\left(b a, \rho_{b}\right)=\frac{(5-\sqrt{5})}{10}, \\
\left(c a, \rho_{b}\right)=\frac{(-5+3 \sqrt{5})}{10}, \quad\left(c b, \rho_{b}\right)=\frac{(5-\sqrt{5})}{10} .
\end{array}
$$

We get $\left(b, \rho_{b}\right)=\left(b a, \rho_{b}\right)$ and $\left(c, \rho_{b}\right)=\left(c a, \rho_{b}\right)+\left(c b, \rho_{b}\right)$ which indicates that additivity holds. One way to calculate the above numbers is to compute the Jordan decomposition of the matrix $\mathcal{B}_{l}$.

Example 5.2. Let $F_{4}=\langle a, b, c, d\rangle$. Let $G$ be the rose on four petals labeled $a, b, c, d$. Consider an outer automorphism $\Phi$ given by a train track representative $\phi: G \rightarrow G$ by

$$
\phi(a)=a b b a b, \phi(b)=b a b a b b a b, \phi(c)=c a d, \phi(d)=d c a d .
$$

Let $\mathcal{A}=\{[\langle a, b\rangle]\}$. The transition matrix for $\phi$ is given by

$$
M=\left[\begin{array}{llll}
c & d & a & b \\
1 & 1 & 0 & 0 \\
1 & 2 & 0 & 0 \\
1 & 1 & 2 & 3 \\
0 & 0 & 3 & 5
\end{array}\right] .
$$

Let $\rho_{c}=\lim _{n \rightarrow \infty} \phi^{n}(c)$. We can view $\phi$ as a substitution on the alphabet $\mathbb{A}=\{a, b, c, d\}$. Let $\mathbb{A}_{l}$ be the set of words of length $l$ on $\mathbb{A}$ that appear in $\rho_{c}$. We want to calculate the frequency of occurrences of words, which cross $c$ and $d$, in $\rho_{c}$. Let $w \in \mathbb{A}_{l}$ and let $\gamma$ be a word of length $l$ that starts with $c$. Using the same notation as in the previous example we have

$$
\lim _{n \rightarrow \infty} \frac{\left(w, \phi^{n}(c)\right)}{\lambda^{n}}=\lim _{n \rightarrow \infty} \frac{M_{l}^{n}(w, \gamma)}{\lambda^{n}}=\lim _{n \rightarrow \infty} \frac{\mathcal{B}_{l}^{n}(w, \gamma)}{\lambda^{n}}=: d_{w, c} .
$$

For example, in length two we have $\mathbb{A}_{2}=\{a b, b a, b b, a d, b d, c a, d a, d c\}$ and $\mathcal{B}_{2}=$ $\{a d, b d, c a, d a, d c\}$. We get the matrices

$$
\mathcal{B}_{1}=\left[\begin{array}{cc}
b & c \\
1 & 1 \\
1 & 2
\end{array}\right], \quad \mathcal{B}_{2}=\left[\begin{array}{ccccc}
1 & 1 & 1 & 0 & 0 \\
1 & 1 & 0 & 0 & 0 \\
0 & 1 & 2 & 0 & 0 \\
1 & 1 & 1 & 0 & 0 \\
0 & 0 & 0 & 1 & 1
\end{array}\right]
$$

and compute the frequencies as in the previous example. 
Example 5.3. Let $F_{4}=\langle a, b, c, d\rangle$. Let $G$ be the rose on four petals labeled $a, b, c, d$. Consider an outer automorphism $\Phi$ given by a train track representative $\phi: G \rightarrow G$ by

$$
\phi(a)=a b, \phi(b)=b a b, \phi(c)=c a d, \phi(d)=d c a d .
$$

Let $\mathcal{A}=\{[\langle a, b\rangle]\}$. The transition matrix for $\phi$ is given by

$$
M=\left[\begin{array}{llll}
c & d & a & b \\
1 & 1 & 0 & 0 \\
1 & 2 & 0 & 0 \\
1 & 1 & 1 & 1 \\
0 & 0 & 1 & 2
\end{array}\right] .
$$

Let $\rho_{c}=\lim _{n \rightarrow \infty} \phi^{n}(c)$. We can view $\phi$ as a substitution on the alphabet $\mathbb{A}=\{a, b, c, d\}$. As before we have

$$
\lim _{n \rightarrow \infty} \frac{\left(w, \phi^{n}(c)\right)}{\lambda^{n}}=\lim _{n \rightarrow \infty} \frac{M_{l}^{n}(w, \gamma)}{\lambda^{n}}=\lim _{n \rightarrow \infty} \frac{\mathcal{B}_{l}^{n}(w, \gamma)}{\lambda^{n}}=: d_{w, c},
$$

where $\lambda$ is the PF-eigenvalue of the top stratum. For length two, we have $\mathbb{A}_{2}=$ $\{a b, b a, b b, a d, b d, c a, d a, d c\}$ and $\mathcal{B}_{2}=\{a d, b d, c a, d a, d c\}$. We get the matrices

$$
\mathcal{B}_{1}=\left[\begin{array}{cc}
b & c \\
1 & 1 \\
1 & 2
\end{array}\right], \quad \mathcal{B}_{2}=\left[\begin{array}{ccccc}
1 & 1 & 1 & 0 & 0 \\
1 & 1 & 0 & 0 & 0 \\
0 & 1 & 2 & 0 & 0 \\
1 & 1 & 1 & 0 & 0 \\
0 & 0 & 0 & 1 & 1
\end{array}\right]
$$

and compute the frequencies as above.

In all the above examples, the topological representatives of the outer automorphisms were defined on roses whose universal covers are Cayley graphs. Thus, to associate a relative current to such outer automorphisms, we can use coordinates coming from the respective Cayley graphs. In general, a topological representative of an outer automorphism is defined on some marked graph in outer space. Thus, before we show how to associate limiting currents to relatively fully irreducible outer automorphims, we define coordinates with respect to a marked graph.

Definition 5.4 (Coordinates with respect to a marked graph). Let $G$ be a marked metric graph in Culler-Vogtmann's outer space, such that $G$ has a subgraph $\Gamma$ with $\mathcal{F}(\Gamma)=\mathcal{A}$. Let $g: \mathcal{R} \rightarrow G$ be the marking of $G$. Here $\mathcal{R}$ is the quotient of $\operatorname{Cay}\left(\mathbb{F}, \mathfrak{B}_{\mathcal{A}}\right)$ under the action of $\mathbb{F}$. Let $\widetilde{G}$ be the universal cover of $G$. The map $g$ lifts to an $\mathbb{F}$-equivariant map $\widetilde{g}: \operatorname{Cay}\left(\mathbb{F}, \mathfrak{B}_{\mathcal{A}}\right) \rightarrow \widetilde{G}$. The map $\widetilde{g}$ identifies $\partial^{2} \widetilde{G}$ with $\partial^{2} \mathbb{F}$ and $\partial^{2} \Gamma$ with $\partial^{2} \mathcal{A}$. Given an edge-path $v$ in $\widetilde{G}$, let

$$
C(v):=\left\{(x, y) \in \partial^{2} \mathbb{F} \mid v \subset(\widetilde{g}(x), \widetilde{g}(x))\right\}
$$

be a compact open set of $\partial^{2} \mathbb{F}$ determined by the subpath $v$ of $\widetilde{G}$. For a relative current $\eta$ and a path $v$ of $\widetilde{G}$ that is not entirely contained in the lift of $\Gamma, \eta(v)$ is defined to be equal to $\eta(C(v))$. Since $\eta$ is $\mathbb{F}$-equivariant, we may consider $v$ to be a reduced edge-path in $G$ itself. The collection of compact open sets $C(v)$ for all paths $v$ in $G$ that are not entirely contained in $\Gamma$ contains the cylinder sets determined by words in $\mathbb{F}$ that determine a basis for topology of $\partial^{2} \mathbb{F}$. Since a relative current is uniquely determined by its values on elements in $\mathbb{F} \mathcal{A}$, it is also uniquely determined 
by its values on compact open sets determined by reduced paths $v$ in $G$ that are not entirely contained in $\Gamma$.

The next lemma defines a limiting current for a relative fully irreducible outer automorphism.

Lemma 5.5. Let $\phi: G \rightarrow G$ be a completely split train track representative of $\Phi$, a fully irreducible outer automorphism relative to $\mathcal{A}$. Let a be an edge in the top $E G$ stratum $H_{r}$ such that $\rho_{a}$ is fixed under $\phi$. Let $v$ be any reduced edge path in $G$ that crosses $H_{r}$. Let $d_{v, a}$ be the frequency of occurrence of $v$ in $\rho_{a}$. Then the set of values

$$
d_{v, a}+d_{\bar{v}, a}=: \eta_{\phi}^{a}(v)
$$

define a unique current $\eta_{\phi}^{a}$ relative to $\mathcal{A}$. That is,

(a) $\eta_{\phi}^{a}(v) \geq 0$,

(b) $\eta_{\phi}^{a}(v)=\eta_{\phi}^{a}(\bar{v})$,

(c) $\eta_{\phi}^{a}(v)=\sum_{e \in E} \eta_{\phi}^{a}(v e)$, where $E$ is the set of edges of $G$ incident at endpoint of $v$ and $e$ is not equal to the inverse of the terminal edge of $v$.

For an edge $b \neq a$ in $H_{r}$, we have that $\eta_{\phi}^{b}=\kappa \eta_{\phi}^{a}$ for some constant $\kappa\left(a, b,\left.\phi\right|_{H_{r}}\right)$. Thus for every fully irreducible outer automorphism relative to $\mathcal{A}$, we get a unique projective relative current, denoted $\left[\eta_{\Phi}^{+}\right]=\left[\eta_{\phi}^{a}\right]$.

Proof. By Proposition 4.1 we know that the values $d_{v, a}$ exist and are non-negative for all reduced paths $v$ in $G$ that cross $H_{r}$. The equation (b) holds by definition of $\eta_{\phi}^{a}(v)$. Proposition 4.1 provides a substitution determined by $\phi$. Applying Proposition 8.12 to this substitution we see that $\eta_{\phi}^{a}(v)$ satisfies Kirchoff's laws, that is, (c) holds. Since a relative current is uniquely determined by its values on compact open sets in $\partial^{2} \mathbb{F}$ determined by reduced paths in $G$ that cross $H_{r}$, we get a unique relative current $\eta_{\phi}^{a}$. Again by Proposition 4.1, we have $\eta_{\phi}^{a}(v)=\kappa \eta_{\phi}^{b}(v)$ for all reduced paths $v$ in $G$ that cross $H_{r}$ and for some constant $\kappa$. Thus the projective class $\left[\eta_{\phi}^{a}\right]=:\left[\eta_{\Phi}^{+}\right]$of the relative current $\eta_{\phi}^{a}$ is also unique.

The projective relative current $\left[\eta_{\Phi}^{+}\right]$is called the stable current for $\Phi$. The stable current for $\Phi^{-1}$, denoted $\left[\eta_{\Phi}^{-}\right]$, is called the unstable current for $\Phi$.

\section{Goodness}

In BFH97, Bestvina, Feighn and Handel studied the legal structure of conjugacy classes under forward and backward iterates of a train track representative of a fully irreducible outer automorphism. In [Bri00], Brinkmann generalized some of those results to relative train track maps which we use in this section.

Throughout this section $\Phi \in \operatorname{Out}(\mathbb{F}, \mathcal{A})$ will be a fully irreducible outer automorphism relative to $\mathcal{A}$ and $\phi: G \rightarrow G$ a completely split train track representative of $\Phi$ with filtration $\emptyset=G_{0} \subset G_{1} \subset \cdots \subset G_{r}=G$ such that $\mathcal{F}\left(G_{r-1}\right)=\mathcal{A}$, and $H_{r}$ is the top EG stratum with $\mathrm{PF}$ eigenvalue $\lambda_{\Phi}>1$. In this section, we use Facts 2.1 about completely split train track maps.

In Bri00, Brinkmann considers the following metric on $G$ : edges in $H_{r}$ get length according to the PF eigenvector for the transition matrix for $H_{r}$, such that the smallest length is one and hence edges in $H_{r}$ get stretched by $\lambda_{\Phi}$ under the application of $\phi$. Edges in $G_{r-1}$ get length one. See Section 2.7. 
Throughout, we use the same notation for a conjugacy class in $[\mathbb{F}]$ and its representative in $G$ which is taken to be cyclically reduced. For a reduced path $\rho$ in $G$, the tightened image of $\rho$ is denoted by $[\phi(\rho)]$. Define $i_{r}(\rho)$ to be the number of $r$-illegal turns in $\rho, l_{r}(\rho)$ the $r$-length of $\rho$ and $L_{r}(\rho)$ the length of the longest $r$-legal segment in $\rho$. Recall from Section 2.7 that $L_{r}^{c}=\frac{2 B C C(\phi)}{\lambda_{\Phi}-1}$ is the critical $r$-length where $B C C(\phi)$ is the bounded cancellation constant.

Denote by $\rho^{-k}$ a path in $G$ with the property that the tightened image of $\phi^{k}\left(\rho^{-k}\right)$ is $\rho$. For a subpath $\rho$ of a path $\sigma$, let $\left[\phi^{k}(\rho)\right]_{\sigma}$ denote the maximal subpath of $\left[\phi^{k}(\rho)\right]$ contained in $\left[\phi^{k}(\sigma)\right]$.

The following proposition is a generalization of [BFH97, Lemma 2.9].

Proposition 6.1 (Bri00, Lemma 6.2]). Let $\phi: G \rightarrow G$ be a relative train track map and let $H_{r}$ be an EG stratum. For every $L>0$, there exists $M(L)>0$ such that if $\rho$ is a path in $G_{r}$ that crosses $H_{r}$, then one of the following holds:

(a) $\left[\phi^{M}(\rho)\right]$ contains an $r$-legal segment of $r$-length $>L$.

(b) $\left[\phi^{M}(\rho)\right]$ has fewer r-illegal turns.

(c) $\rho$ can be expressed as a concatenation $\tau_{1} \rho^{\prime} \tau_{2}$, where $l_{r}\left(\tau_{1}\right) \leq 2 L, l_{r}\left(\tau_{2}\right) \leq$ $2 L, i_{r}\left(\tau_{1}\right) \leq 1, i_{r}\left(\tau_{2}\right) \leq 1$, and $\rho^{\prime}$ splits as a concatenation of pre-Nielsen paths (with one $r$-illegal turn each) and segments in $G_{r-1}$.

Lemma 6.2 (Backward iterations). Let $\phi: G \rightarrow G$ be a completely split train track representative of a fully irreducible outer automorphism relative to $\mathcal{A}$. Given some number $L_{0}>0$, there exists $M>0$, depending only on $L_{0}$ and $H_{r}$, such that for any subpath $\rho$ of an $\mathcal{A}$-separable conjugacy class $\alpha$ realized in $G_{r}$ with $1 \leq L_{r}(\rho) \leq L_{0}$ and $i_{r}(\rho) \geq 5$, we have

$$
\left(\frac{10}{9}\right)^{n} i_{r}(\rho) \leq i_{r}\left(\rho^{-n M}\right)
$$

for all $n>0$.

Proof. In [Bri00, Lemma 6.4], Brinkmann proves the same statement for atoroidal outer automorphisms and for any path in $G_{r}$. The same proof follows by using Facts 2.1 about completely split train track representatives.

Given $L=L_{0}+L_{r}^{c}$, choose $M$ as in Proposition 6.1. Subdivide the path $\rho$ into subpaths $\rho_{1}, \ldots, \rho_{m}, \tau$ such that $i_{r}\left(\rho_{i}\right)=5$ and $i_{r}(\tau)<5$. Let $\rho_{i}^{-M}$ be the preimage of $\rho_{i}$ under $\phi^{M}$. Then $\rho^{-M}$ is the concatenation of $\rho_{i}^{-M}$ and $\tau^{-M}$. We claim that $i_{r}\left(\rho_{i}^{-M}\right) \geq 6$ for all $i$. Suppose for contradiction that $i_{r}\left(\rho_{i}^{-M}\right)=5$ for some $i$. Then by Proposition 6.1, $\rho_{i}^{-M}$ splits as a concatenation of at least three pre-Nielsen paths and paths in $G_{r-1}$. By Facts 2.1, every Nielsen path has period one and there is at most one INP $\sigma$ of height $r$. If $\sigma$ is not closed, then at least one endpoint of $\sigma$ is not contained in $G_{r-1}$. Therefore, we cannot have three Nielsen paths in $\rho_{i}^{-M}$ separated by paths in $G_{r-1}$. If $\sigma$ is closed, then its endpoint is not in $G_{r-1}$. Since $\alpha$ is $\mathcal{A}$-separable, it cannot have two consecutive occurrences of $\sigma$ in it. Indeed, since $\sigma$ (which is not contained in $G_{r-1}$ ) is fixed by $\phi$, it is not $\mathcal{A}$-separable. Therefore, its relative Whitehead graph is connected without cut points. If $\alpha$ has two consecutive occurrences of $\sigma$, then its relative Whitehead graph will also be connected without cut points but $\alpha$ is $\mathcal{A}$-separable. Therefore, $\rho$ and $\rho_{i}^{-M}$ cannot have two consecutive occurrences of $\sigma$.

Thus $i_{r}\left(\rho^{-M}\right) \geq 6 m+i_{r}(\tau) \geq(10 / 9) i_{r}(\rho)$ and the lemma follows by induction. 
Lemma 6.3 ([Bri00, Lemma 6.5]). Suppose $H_{r}$ is an EG stratum. Given some $L>0$, there exists some constant $C>0$ such that for all paths $\rho \subset G_{r}$ with $1 \leq L_{r}(\rho) \leq L$ and $i_{r}(\rho)>0$, we have

$$
C^{-1} i_{r}(\rho) \leq l_{r}(\rho) \leq C i_{r}(\rho) .
$$

The notion of goodness was introduced in [Mar95] and formalized in BFH97.

Definition 6.4 (Goodness). Given a loop or a path $\alpha$ in $G_{r}$ that crosses $H_{r}$, the good portion, denoted $g$, of $\alpha$ is the set of $r$-legal segments that are $r$-distance $L_{r}^{c}$ away from $r$-illegal turns. The bad portion, denoted $b$, is the part of $\alpha$ which is $r$-distance less than equal to $L_{r}^{c}$ from an $r$-illegal turn. The $r$-length of $\alpha$ is equal to the $r$-length of $g$ (denoted $\left.g_{r}(\alpha)\right)$ plus the $r$-length of $b$ (denoted $b_{r}(\alpha)$ ). Goodness of $\alpha$ is defined as

$$
\mathfrak{g}(\alpha)=\frac{g_{r}(\alpha)}{l_{r}(\alpha)} .
$$

Lemma 6.5. Let $\delta>0$ and $\epsilon>0$ be given. Then there exists an integer $M=$ $M(\delta, \epsilon)$ such that for any $\mathcal{A}$-separable conjugacy class $\alpha$ that crosses $H_{r}$ with $\mathfrak{g}(\alpha) \geq$ $\delta$, we have $\mathfrak{g}\left(\phi^{m}(\alpha)\right) \geq 1-\epsilon$ for all $m \geq M$.

The proof of the above lemma which is the same as in the absolute case can be found in Uya14, Lemma 3.10]

Definition 6.6 (Desired growth Bri00). Let $\sigma$ be a path in $G$ that crosses an EG stratum $H_{r}$. We say $\sigma$ has desired growth if there exist $N>0, \lambda>1, \epsilon>0$ and a collection of subpaths $S$ of $\sigma$ such that the following hold:

(a) For every integer $n>0$ and for every $\rho \in S$, we have

$$
\lambda^{n} l_{r}(\rho) \leq \max \left\{l_{r}\left(\left[\phi^{n N}(\rho)\right]_{\sigma}\right), l_{r}(\gamma)\right\},
$$

where $\gamma$ is a subpath of $\sigma^{-n N}$ such that $\left[\phi^{n N}(\gamma)\right]_{\sigma^{-n N}}=\rho$.

(b) There is no overlap between distinct paths in $S$.

(c) The sum of the lengths of the paths in $S$ is at least $\epsilon l_{r}(\sigma)$.

Lemma 6.7. Let $\alpha \in[\mathbb{F}]$ be an $\mathcal{A}$-separable conjugacy class that crosses $H_{r}$. Then $\alpha$ has desired growth either under forward iteration or under backward iteration.

Proof. Let $L_{0}>L_{r}^{c}$ be a constant. There are several cases to consider.

(1) $\frac{l_{r}(\alpha)}{i_{r}(\alpha)} \geq L_{0}$. The proof of [Bri00, Proposition $\left.7.1(2)(\mathrm{b})(\mathrm{i})\right]$ shows that in this case $\alpha$ has desired growth in the forward direction.

(2) $\frac{l_{r}(\alpha)}{i_{r}(\alpha)}<L_{0}$

(a) $i_{r}(\alpha) \geq 5$. By [Bri00, Proposition 7.1 (2)(b)(ii)] and using Lemmas $6.2,6.3$ we get desired growth in the backward direction.

(b) $i_{r}(\alpha)<5$. We have that $\alpha$ is $\mathcal{A}$-separable and crosses $H_{r}$ non-trivially. Therefore, $\alpha$ is not fixed and does not have two consecutive occurrences of a closed INP. Since $l_{r}(\alpha)$ is bounded from above, there are only finitely many possibilities for $\alpha \cap H_{r}$. Suppose the $r$-length of no segment of $\alpha \cap H_{r}$ grows under $\phi$. Since there are only finitely many segments of $H_{r}$ of bounded length, after passing to a power we can assume that a segment $\alpha_{i}$ of $\alpha \cap H_{r}$ is fixed under $\phi$. Also the endpoints of $\alpha_{i}$ are in $H_{r} \cap G_{r-1}$. There has to be an illegal turn in $\alpha_{i}$ otherwise 
it would grow and in fact it has to be an INP because it persists. But at least one endpoint of an INP in $G$ is not in $G_{r-1}$, thus we get a contradiction. Therefore we can pass to a uniform power $M$ such that $\phi^{M}(\alpha)$ satisfies (1) and hence has desired growth in forward direction.

It can be seen in Brinkmann's proofs that the numbers $N, \lambda, \epsilon$ do not depend on a specific conjugacy class.

Let $\phi^{\prime}: G^{\prime} \rightarrow G^{\prime}$ be a completely split train track representative of $\Phi^{-1}$. Let $l_{r^{\prime}}$, $i_{r^{\prime}}, L_{r^{\prime}}^{c}$ and $C^{\prime}$ be the corresponding notation related to $\phi^{\prime}$. There exists a constant $B$ such that for any conjugacy class $\alpha$ we have

$$
\frac{l_{r^{\prime}}(\alpha)}{B} \leq l_{r}(\alpha) \leq B l_{r^{\prime}}(\alpha)
$$

Let $\mathfrak{g}^{\prime}$ denote the goodness with respect to the train track structure of $\phi^{\prime}$.

Lemma 6.8. Given $\delta>0$, there exists $M>0$ such that for any $\mathcal{A}$-separable conjugacy class $\alpha$ that crosses $H_{r}$ either

- $\mathfrak{g}\left(\phi^{n M}(\alpha)\right) \geq \delta$ for all $n \geq 1$ or

- $\mathfrak{g}^{\prime}\left(\left(\phi^{\prime}\right)^{n M}(\alpha)\right) \geq \delta$ for all $n \geq 1$.

Proof. Let $L_{0}>L_{r}^{c}$ be the constant from Lemma 6.7 By the same lemma, there exist $N>0, \lambda>1$ and $\epsilon>0$ such that any $\mathcal{A}$-separable conjugacy class that crosses $H_{r}$ has desired growth. There are two cases:

(a) Let us first consider the case when $\alpha$ has desired growth in the forward direction. This happens when $l_{r}(\alpha) \geq L_{0} i_{r}(\alpha)$. For case 2(b) in the proof of Lemma 6.7 we pass to a uniform power of $\alpha$ which satisfies $l_{r}(\alpha) \geq L_{0} i_{r}(\alpha)$. Let $S$ be the collection of maximal $r$-legal subpaths of $\alpha$ of $r$-length at least $L_{0}+1$. Then by the choice of $L_{0}$ we have for $\rho \in S$,

$$
l_{r}\left(\phi^{n N}(\rho)\right) \geq \lambda_{\Phi}^{n N} \frac{1}{L_{0}+1} l_{r}(\rho) .
$$

We have that the paths in $S$ account for a definite fraction $\epsilon>0$ of $\alpha$. Now

$$
g_{r}\left(\phi^{n N}(\alpha)\right) \geq \sum_{\rho \in S}\left[l_{r}\left(\phi^{n N}(\rho)\right)\right]_{\alpha} \geq \sum_{\rho \in S} \lambda_{\Phi}^{n N} \frac{1}{L_{0}+1}\left[l_{r}(\rho)\right]_{\alpha} \geq \lambda_{\Phi}^{n N} \frac{1}{L_{0}+1} \epsilon l_{r}(\alpha) .
$$

We also have $l_{r}\left(\phi^{n N}(\alpha)\right) \leq \lambda_{\Phi}^{n N} l_{r}(\alpha)$. Thus we get

$$
\mathfrak{g}\left(\phi^{n N}(\alpha)\right) \geq \frac{\epsilon}{L_{0}+1} .
$$

(b) If $\alpha$ has desired growth in the backward direction, then by Lemma 6.2 and

Lemma 6.3, we have

$$
B l_{r^{\prime}}\left(\left(\phi^{\prime}\right)^{n N}(\alpha)\right) \geq l_{r}\left(\phi^{-n N}(\alpha)\right) \geq C^{-1} i_{r}\left(\phi^{-n N}(\alpha)\right) \geq\left(\frac{10}{9}\right)^{n} \frac{1}{C^{2} B} l_{r^{\prime}}(\alpha) .
$$

Now the number of $r^{\prime}$-illegal turns in $\left(\phi^{\prime}\right)^{n N}(\alpha)$ is bounded above by those in $\alpha$. We have

$$
i_{r^{\prime}}\left(\left(\phi^{\prime}\right)^{n N}(\alpha)\right) \leq i_{r^{\prime}}(\alpha) \leq C^{\prime} l_{r^{\prime}}(\alpha) .
$$

Also the bad portion of $\left(\phi^{\prime}\right)^{n N}(\alpha)$ is bounded from above by $2 L_{r^{\prime}}^{c} i_{r^{\prime}}\left(\left(\phi^{\prime}\right)^{n N}(\alpha)\right)$. Thus

$$
\mathfrak{g}^{\prime}\left(\left(\phi^{\prime}\right)^{n N}(\alpha)\right) \geq 1-\frac{2 L_{r^{\prime}}^{c} C^{\prime} B^{2} C^{2}}{(10 / 9)^{n}} \geq 1-\frac{2 L_{r^{\prime}}^{c} C^{\prime} B^{2} C^{2}}{(10 / 9)} .
$$


Now by Lemma 6.5, we find $M>0$ such that either one of the goodness is greater than $\delta$.

\section{NORTH-SOUTH DYNAMICS}

We are now ready to prove a north-south dynamic result. Recall $\Phi$ is a fully irreducible outer automorphism relative to $\mathcal{A}$ and $\phi: G \rightarrow G$ is a completely split train track representative of $\Phi$. We also have a stable current $\left[\eta_{\Phi}^{+}\right]$and an unstable current $\left[\eta_{\Phi}^{-}\right]$in $\mathcal{M R C}(\mathcal{A})$.

Proposition 7.1. Given a neighborhood $U$ of $\left[\eta_{\Phi}^{+}\right]$in $\mathcal{M R C}(\mathcal{A})$, there exists $0<$ $\delta<1$ and $M(U)>0$ such that for any $\left[\eta_{\alpha}\right] \in \mathcal{M R C}(\mathcal{A})$, with $\mathfrak{g}(\alpha)>\delta$, we have that $\phi^{n}\left(\left[\eta_{\alpha}\right]\right) \in U$ for all $n \geq M$.

The proof of the above lemma is similar to the proof of [Uya14, Lemma 3.11].

Lemma 7.2. Given neighborhoods $U$ and $V$ of $\left[\eta_{\Phi}^{+}\right]$and $\left[\eta_{\Phi}^{-}\right]$in $\mathcal{M R C}(\mathcal{A})$, respectively, there exists $M_{1}>0$ such that for any $\mathcal{A}$-separable conjugacy class $\alpha$ that crosses $H_{r}$ either $\phi^{m}\left(\left[\eta_{\alpha}\right]\right) \in U$ or $\left(\phi^{\prime}\right)^{m}\left(\left[\eta_{\alpha}\right]\right) \in V$ for all $m \geq M_{1}$.

The proof follows from Lemma 6.8 and Lemma 7.1

Proposition 7.3 ([LU15, Proposition 3.4]). Let $\phi: X \rightarrow X$ be a homeomorphism of a compact space $X$ and assume that $X$ is sufficiently separable, for example, metrizable. Let $Y \subset X$ be a dense set, and let $\mathcal{P}, \mathcal{Q}$ be two distinct $\phi$-invariant points in $X$. Assume the following holds: for every neighborhood $U$ of $\mathcal{P}$ and $V$ of $\mathcal{Q}$, there exists an integer $M_{2} \geq 1$ such that for all $m \geq M_{2}$ and all $y \in Y$ one has either $\phi^{m}(y) \in U$ or $\phi^{-m}(y) \in V$. Then $\phi^{2}$ has uniform north-south dynamics from $\mathcal{P}$ to $\mathcal{Q}$.

Proposition 7.4 ([LU15, Proposition 3.5]). Let $\phi: X \rightarrow X$ be as in Proposition 7.3 with distinct fixed points $\mathcal{P}$ and $\mathcal{Q}$ and assume that some power $\phi^{s}$ with $s \geq 1$ has uniform north-south dynamics from $\mathcal{P}$ to $\mathcal{Q}$. Then $\phi$ also has uniform north-south dynamics from $\mathcal{P}$ to $\mathcal{Q}$.

Theorem A. Let $\mathcal{A}$ be a non-trivial free factor system of $\mathbb{F}$ such that $\zeta(\mathcal{A}) \geq 3$. Let $\Phi \in \operatorname{Out}(\mathbb{F}, \mathcal{A})$ be fully irreducible relative to $\mathcal{A}$. Then $\Phi$ acts with uniform north-south dynamics on $\mathcal{M R C}(\mathcal{A})$.

Proof. The proof follows from Lemma 7.2, Proposition 7.3 and Proposition 7.4 .

\section{Appendix}

8.1. Extension of relative currents. In this section we will prove Lemma 3.15, which says that given a relative current $\eta_{0}$ there exists a signed measured current $\eta$ which is a $k$-extension of $\eta_{0}$. We will first show that $\eta_{0}$ can be extended to a signed measured current $\eta$ which may or may not be non-negative on all words of length less than or equal to $k$. We then show how to modify $\eta$ to get a $k$-extension of $\eta_{0}$.

Remark 8.1. Throughout this section we will assume that $\mathcal{A}$ has only one conjugacy class of a free factor $\left[A_{0}\right]$. When $\mathcal{A}$ has more than one free factor in it then the same process can be repeated for all the free factors independently of each other. 


\section{Notation:}

- Let $\mathfrak{B}_{\mathcal{A}}$ be a relative basis of $\mathbb{F}$. Let $s$ be the rank of the free factor $A_{0}$. Denote the generators of $A_{0}$ by $a_{i}, 1 \leq i \leq s$. Also let $A:=\left\{a_{1}^{ \pm}, \ldots, a_{s}^{ \pm}\right\}$.

- Let $S_{k}$ be the set of words in $A_{0}$ of length $k$ with respect to $\mathfrak{B}_{\mathcal{A}}$. Let \# $S_{k}$ denote the cardinality of $S_{k}$.

- Let $S_{k}^{0}$ be a subset of $S_{k}$ (chosen once and for all) such that for every $w \in S_{k}$ exactly one of $w$ or $\bar{w}$ appears in $S_{k}^{0}$.

- The letters $e, x, y, z$ will denote elements of $\mathfrak{B}_{\mathcal{A}}$.

- Whenever a forward (backward) extension of a word $w$ by $e \in \mathfrak{B}_{\mathcal{A}}$ is written as $w e(e w)$ it is to be understood that $e$ is not the inverse of the last (first) letter of $w$.

For every $k>0$ we will define a signed measured current $\eta$ on words in $A_{0}$ of length $(k-1)$ and use those values together with the additivity laws satisfied by $\eta$ to define $\eta$ on words of length $k$. To start with words of length one, choose arbitrary values for $\eta\left(a_{i}\right)$ for all $1 \leq i \leq s$. By induction assume $\eta(v)$ is defined for all words $v$ of length less than equal to $(k-1)$. By additivity, for all $v \in S_{k-1}^{0}$ the following hold:

$$
\begin{aligned}
& \eta(v)=\sum_{e \in A} \eta(v e)+\sum_{e \notin A} \eta_{0}(v e), \\
& \eta(\bar{v})=\sum_{e \in A} \eta(\bar{v} e)+\sum_{e \notin A} \eta_{0}(\bar{v} e) .
\end{aligned}
$$

Since $\eta$ is invariant under taking inverses, the equation obtained from forward extension of $\bar{v}$ is the same as the equation obtained from backward extension of $v$.

Rearranging the equations to have the unknown terms on the left-hand side we get

$$
\begin{aligned}
& \sum_{e \in A} \eta(v e)=\eta(v)-\sum_{e \notin A} \eta_{0}(v e)=c_{v}, \\
& \sum_{e \in A} \eta(\bar{v} e)=\eta(\bar{v})-\sum_{e \notin A} \eta_{0}(\bar{v} e)=c_{\bar{v}} .
\end{aligned}
$$

Thus there are $\# S_{k-1}$ equations in $\# S_{k}^{0}$ variables and the number of variables are more than the number of equations. Denote this system of equations by $E_{k-1}^{1}$, that is, equations obtained from one edge extensions of length $(k-1)$ words. Similarly we can look at the system $E_{k-i}^{i}$.

Consider the augmented matrix $[M \mid c]$ for the system of equations $E_{k-1}^{1}$ with rows labeled by $v \in S_{k-1}$ and columns by $w \in S_{k}^{0}$. If $w=v e$ or $\bar{w}=v e$ for some $e \in A$, then $M_{v, w}=1$, otherwise, $M_{v, w}=0$. We will denote a row vector of $M$ by $r_{v}$ corresponding to $v \in S_{k-1}$. We make some observations about the matrix $M$.

- Each column has exactly two 1's. Indeed, $M_{v, w}$ is 1 exactly when $v$ is a prefix of $w$ or $\bar{w}$.

- There are $(2 s-1)$ non-zero entries in each row because there are $(2 s-1)$ possible extensions of $v$ by $e \in A$.

- Any two distinct rows can be same in at most one column. Let $w$ be common to two distinct rows $r_{v_{1}}$ and $r_{v_{2}}$. Then

$$
w=v_{1} e_{1} \text { or } \overline{e_{1}} \overline{v_{1}} \quad \text { and } \quad w=v_{2} e_{2} \text { or } \overline{e_{2}} \overline{v_{2}}
$$


for some $e_{1}, e_{2} \in A$. Then it must be true that $v_{1}$ begins with $\overline{e_{2}}$ and $v_{2}$ begins with $\overline{e_{1}}$. Thus $w$ is uniquely determined.

\section{Lemma 8.2.}

(a) For every $i \geq 1$, an equation in the system $E_{k-i-1}^{i+1}$ is a linear combination of equations in the system $E_{k-i}^{i}$. Thus it is sufficient to look at the system $E_{k-1}^{1}$ to obtain all constraints satisfied by $\eta(w)$ for all $w \in S_{k}^{0}$.

(b) Let $u \in S_{k-2}$. Then we have

$$
\sum_{x \in A} r_{x u}=\sum_{x \in A} r_{x \bar{u}}
$$

(c) The set of relations $\sum_{x \in A} r_{x u}=\sum_{x \in A} r_{x \bar{u}}$ for every $u \in S_{k-2}$ generate any other relation among the rows of $M$.

(d) We also have

$$
\sum_{x \in A} c_{x u}=\sum_{x \in A} c_{x \bar{u}}
$$

where $c_{v}$ is the constant term of the equation determined by $v \in S_{k-1}$.

(e) The system of equations $E_{k-1}^{1}$ is consistent and hence has a solution. Thus we can define $\eta$ on words of length $k$.

Proof. $\quad$ (a) Let $u \in S_{k-i-1}$. Then

$$
\eta(u)=\sum_{x \in A} \eta(u x)+\sum_{x \notin A} \eta(u x) .
$$

By equations in $E_{k-i}^{i}$, we have

$$
\eta(u x)=\sum_{y \in \mathbb{F},|y|=i} \eta(u x y) .
$$

Adding all these equations over $x \in \mathfrak{B}_{\mathcal{A}}$ we get

$$
\eta(u)=\sum_{x, y \in \mathbb{F},|x|=1,|y|=i} \eta(u x y)=\sum_{z \in \mathbb{F},|z|=i+1} \eta(u z) .
$$

Thus we recovered an equation in $E_{k-i-1}^{i+1}$ by a combination of equations in $E_{k-i}^{i}$.

(b) For every $x \in A, M_{x u, w} \neq 0$ exactly when $w=x u \bar{y}$ or $w=y \bar{u} \bar{x}$ for some $y \in A$. Therefore, if $M_{x u, w} \neq 0$, then $M_{y \bar{u}, w} \neq 0$ for some $y \in A$.

(c) Consider a minimal relation $R$ given by $\sum_{v \in S_{k-1}} d_{v} r_{v}=0$, where $d_{v} \in \mathbb{R}$. We can rescale the equation such that coefficient of at least one row, say $r_{x u}$ for some $x \in A$ and $u \in S_{k-2}$, is 1 .

For every $y \in A$ and $w=x u \bar{y}$, we have $M_{x u, w}=M_{y \bar{u}, w}=1$. Thus $r_{x u}$ and $r_{y \bar{u}}$ share exactly one common entry $w$ and no other row has a non-zero entry in $w$. Thus $d_{y \bar{u}}=-1$. Now consider $y \in A$. For any $z \in A$ and $w=y \bar{u} z$, we have $M_{y \bar{u}, w}=M_{\bar{z} u, w}=1$. Thus $d_{\bar{z} u}=1$. Hence our minimal relation is just $\sum_{x \in A} r_{x u}-\sum_{y \in A} r_{y \bar{u}}=0$. 
(d) We have

$$
\begin{aligned}
\sum_{x \in A} c_{x u} & =\sum_{x \in A} \eta(x u)-\sum_{x \in A, y \notin A} \eta(x u y) \\
& =\eta(u)-\sum_{x \notin A} \eta(x u)-\sum_{x \in A, y \notin A} \eta(x u y) \\
& =\eta(u)-\sum_{x \notin A, y \in \mathfrak{B}_{\mathcal{A}}} \eta(x u y)-\sum_{x \in A, y \notin A} \eta(x u y)
\end{aligned}
$$

and, similarly,

$$
\begin{aligned}
\sum_{x \in A} c_{x \bar{u}} & =\eta(u)-\sum_{x \notin A, y \in \mathfrak{B}_{\mathcal{A}}} \eta(x \bar{u} y)-\sum_{x \in A, y \notin A} \eta(x \bar{u} y) \\
& =\eta(u)-\sum_{x \notin A, y \in \mathfrak{B}_{\mathcal{A}}} \eta(\bar{y} u \bar{x})-\sum_{x \in A, y \notin A} \eta(\bar{y} u \bar{x}) .
\end{aligned}
$$

We see that

$$
\sum_{x \notin A, y \in \mathfrak{B}_{\mathcal{A}}} \eta(x u y)+\sum_{x \in A, y \notin A} \eta(x u y)=\sum_{x \notin A, y \in \mathfrak{B}_{\mathcal{A}}} \eta(\bar{y} u \bar{x})+\sum_{x \in A, y \notin A} \eta(\bar{y} u \bar{x}) .
$$

Geometrically, we are looking at the same subset of $\partial^{2} \mathbb{F}$ as a union of cylinder sets in two different ways. See Figure 1 when $\mathbb{F}=\langle a, b, c, d\rangle$.

$$
u=a
$$

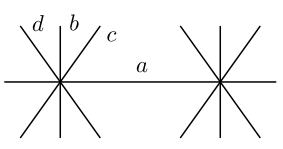

LHS
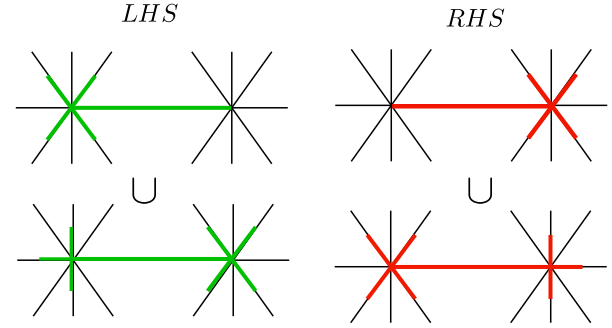

FIGURE 1

(e) Since the relations which generate all other relations among the rows of $M$ are consistent, $[M \mid c]$ has a solution.

Proof of Lemma 3.15. Given a relative current $\eta_{0}$, by Lemma 8.2, we can find a signed measured current $\eta$ such that $\eta_{0}(w)=\eta(w)$ for all $w \in \mathbb{F} \backslash \mathcal{A}$. This extension need not be non-negative on all words of length less than or equal to $k$. Let $-M$ for $M>0$ be the smallest value attained by $\eta(w)$ for a word $w \in \mathcal{A}$ with $|w| \leq k$. Consider a signed measured current $\eta_{\mathcal{A}, C}$ defined as follows:

$$
\eta_{\mathcal{A}, C}(w)=\frac{C}{(2 s-1)^{|w|-1}} \text { for } w \in \mathcal{A} \text { and } 0 \text { otherwise. }
$$


For $C=M(2 s-1)^{k-1}, \eta+\eta_{\mathcal{A}, C}$ is non-negative on words of length less than or equal to $k$.

8.2. Substitution dynamics. Let $\mathbb{A}$ be a finite set with cardinality greater than equal to two. Let $\zeta$ be a substitution on $\mathbb{A}$, that is, a map from $\mathbb{A}$ to the set of non-empty words on $\mathbb{A}$ which associates to a letter $e \in \mathbb{A}$ the word $\zeta(e)$ with length $|\zeta(e)|$. The substitution $\zeta$ induces a map on the set of all words on $\mathbb{A}$ by concatenation, that is,

$$
\zeta\left(x_{1} x_{2} \ldots x_{m}\right)=\zeta\left(x_{1}\right) \zeta\left(x_{2}\right) \ldots \zeta\left(x_{m}\right),
$$

where $x_{1} x_{2} \ldots x_{m}$ is a word on $\mathbb{A}$. Thus we can define iterates $\zeta^{n}$ for all $n \geq 1$. To the substitution $\zeta$ we associate its transition matrix, denoted $M$, where for $a, b \in \mathbb{A}$, $M(a, b)$ is the number of occurrence of $a$ in $\zeta(b)$. The transition matrix for $\zeta^{n}$ is given by $M^{n}$. Likewise, we define a map from $\mathbb{A}^{\mathbb{N}}$ to $\mathbb{A}^{\mathbb{N}}$, the set of all infinite words on $\mathbb{A}$, also denoted $\zeta$, by the formula $\zeta\left(x_{1} x_{2} \ldots\right)=\zeta\left(x_{1}\right) \zeta\left(x_{2}\right) \ldots$

Suppose $\zeta$ admits a fixed point, denoted $\rho \in \mathbb{A}^{\mathbb{N}}$, such that $\zeta^{k}(\rho)=\rho$ for all $k \geq 1$. From now on we only keep in the alphabet $\mathbb{A}$ the letters that actually appear in $\rho$.

For every $l>0$, let $\mathbb{A}_{l}$ denote the set of all words on $\mathbb{A}$ of length $l$ that appear in $\rho$. Define a substitution $\zeta_{l}$ on $\mathbb{A}_{l}$ as follows: let $w=x_{1} x_{2} \ldots x_{l} \in \mathbb{A}_{l}$. Define $\zeta_{l}(w):=w_{1} w_{2} \ldots w_{\left|\zeta\left(x_{1}\right)\right|}$ where $w_{i} \in \mathbb{A}_{l}$ and $w_{i}$ is the length $l$ subword of $\zeta(w)$ starting at the $i$ th position of $\zeta\left(x_{1}\right)$. In other words, $\zeta_{l}(w)$ consists of the ordered list of the first $\left|\zeta\left(x_{1}\right)\right|$ subwords of length $l$ of the word $\zeta(w)$. The substitution $\zeta_{l}$ extends to a map on the set of all words on $\mathbb{A}_{l}$. Denote by $|\cdot|_{l}$ the length of words on $\mathbb{A}_{l}$. We have $\left|\zeta_{l}(w)\right|_{l}=\left|\zeta\left(x_{1}\right)\right|$. Denote by $M_{l}$ the transition matrix for $\zeta_{l}$. It is clear from definitions that $\left(\zeta^{n}\right)_{l}=\left(\zeta_{l}\right)^{n}$.

A substitution is called irreducible if for every pair $a, b \in \mathbb{A}$ there exists $k:=$ $k(a, b)$ such that $a$ occurs in $\zeta^{k}(b)$. A substitution is called primitive if there exists $k$ such that for every pair $a, b \in \mathbb{A}, a$ occurs in $\zeta^{k}(b)$.

We are interested in understanding the frequency of occurrence of words on $\mathbb{A}$ that occur in a fixed infinite word $\rho$. In Que87, a theory for understanding these frequencies for a primitive substitution is developed. We want to generalize the theory of primitive substitutions to substitutions which may not be primitive but are primitive on a subset of the alphabet.

8.2.1. Eigenvalues for $M$ and $M_{l}$. The main result from this section is Proposition 8.5. Consider an alphabet $\mathbb{A}=\bigsqcup_{i=0}^{k} B_{i}$. We define a partial order on the alphabet as follows. First define a partial order on subsets of $\mathbb{A}$ given by $B_{i}>B_{j}$ for $i<j$. For example, $B_{0}>B_{1}$ and so on. Thus we get a partial ordering on the letters of $\mathbb{A}$ where $a>b$ if $a \in B_{i}$ and $b \in B_{j}$ where $i<j$. The alphabet $\mathbb{A}_{l}$ can now be given a partial lexicographic order as well. We will consider a substitution $\zeta$ on $\mathbb{A}$ with the following properties:

- For $a \in B_{i}, \zeta(a)$ contains letters only from $B_{j}$ for $j \geq i$. This implies that the transition matrix $M$ for $\zeta$ is lower triangular block diagonal with respect to the partial order on the set $\left\{B_{i}\right\}_{i=0}^{k}$. Denote the diagonal blocks of $M$ also by $B_{i}$ for $0 \leq i \leq k$, where $B_{0}$ is the top left block, followed by $B_{1}$ and so on.

- If $B_{i}$ is a primitive block, then $\zeta(a)$ for $a \in B_{i}$ ends and begins in a letter in $B_{i}$.

- $B_{0}$ is primitive. 
Lemma 8.3. Let $B_{i}$ be a primitive block of $M$. After possibly passing to a power of $\zeta$, there exists $a \in B_{i}$ such that $\zeta(a)$ begins in a. Also, $\rho_{a}:=\lim _{n \rightarrow \infty} \zeta^{n}(a)$ is fixed by $\zeta$, that is, $\zeta\left(\rho_{a}\right)=\rho_{a}$. If $b \in B_{i}$ is another letter which begins in $b$ and $\rho_{b}$ is fixed by $\zeta$, then the set of subwords of $\rho_{a}$ and $\rho_{b}$ are the same.

Proof. Consider a function $f: B_{i} \rightarrow B_{i}$ where for $a \in B_{i}, f(a)$ is the first letter of $\zeta(a)$. Since $B_{i}$ is a finite set, some power of $f$ has a fixed point. After possibly passing to a power, let $a \in B_{i}$ be a fixed point of $f$. Since $\zeta(a)$ begins with $a$, we have that $\zeta^{n}(a)$ begins with $\zeta^{n-1}(a)$ for every $n>0$. Thus $\rho_{a}$ is fixed by $\zeta$. Since $B_{i}$ is a primitive block, $\zeta^{m}(a)$ contains $b$ for some $n>0$. Thus subwords that appear in $\rho_{b}$ also appear in $\rho_{a}$ and vice versa.

We say a word $w$ on $\mathbb{A}$ crosses $B_{i}$ if $w$ contains a letter in $B_{i}$. We are interested in understanding the frequency of occurrence of words in $\rho:=\rho_{0}$ which cross $B_{0}$.

Example 8.4. Let $\mathbb{A}=\{a, b, c, d\}$. Let $\zeta$ be given as $\zeta(a)=a b b a b, \zeta(b)=$ bababbab, $\zeta(c)=c a d, \zeta(d)=d c a d$. The transition matrix for $\zeta$ and $\zeta_{2}$ are given by

$$
M=\left[\begin{array}{cccc}
c & d & a & b \\
1 & 1 & 0 & 0 \\
1 & 2 & 0 & 0 \\
1 & 1 & 2 & 3 \\
0 & 0 & 3 & 5
\end{array}\right], \quad M_{2}=\left[\begin{array}{ccccccccc}
c a & d a & d c & a d & b d & a b & b a & b b \\
1 & 1 & 1 & 0 & 0 & 0 & 0 & 0 \\
1 & 1 & 0 & 0 & 0 & 0 & 0 & 0 \\
0 & 1 & 2 & 0 & 0 & 0 & 0 & 0 \\
0 & 1 & 1 & 0 & 0 & 0 & 0 & 0 \\
0 & 0 & 0 & 1 & 1 & 0 & 0 & 0 \\
0 & 0 & 0 & 2 & 3 & 2 & 3 & 3 \\
0 & 0 & 0 & 1 & 3 & 1 & 4 & 0 \\
0 & 0 & 0 & 1 & 1 & 2 & 1 & 2
\end{array}\right] .
$$

We now want to understand the spectrum of $M_{l}$.

Proposition 8.5. For every $l \geq 2$, the eigenvalues of $M_{l}$ are those of $M$ with possibly some additional eigenvalues of absolute value less than or equal to one.

The three lemmas that follow will be used to prove Proposition 8.5. Since $\left(\zeta^{n}\right)_{l}=$ $\left(\zeta_{l}\right)^{n}$, we have $\left(M^{n}\right)_{l}=\left(M_{l}\right)^{n}$, which we now denote by $M_{l}^{n}$ unless the order needs to be specified. Denote the rows and columns of $M$ by $R_{x}$ and $C_{x}$ for $x \in \mathbb{A}$, those of $M_{l}$ by $R_{w}$ and $C_{w}$ and those of $M_{l}^{n}$ by $R_{n, w}$ and $C_{n, w}$ for $w \in \mathbb{A}_{l}$.

Lemma 8.6. Let $n \geq 2$. Let $M, M_{l}, M_{l}^{n}$ be transition matrices for $\zeta, \zeta_{l}, \zeta_{l}^{n}$, respectively. Then:

(a) $M_{l}$ is a lower triangular block diagonal matrix with respect to the partial order on $\mathbb{A}_{l}$.

(b) Let $w \in \mathbb{A}_{l}$ start with $x \in \mathbb{A}$. Then the sum of the entries of $C_{w}$ is the same as the sum of the entries of $C_{x}$ which is equal to $|\zeta(x)|$.

(c) Let $w_{1}, w_{2} \in \mathbb{A}_{l}$ be such that both words begin with $x \in \mathbb{A}$. Then the entries of $C_{w_{1}}$ and $C_{w_{2}}$ differ at most by $(l-1)$. The entries of $C_{n, w_{1}}$ and $C_{n, w_{2}}$ also differ at most by $(l-1)$.

Proof.

(a) Clear from definitions of $M$ and $M_{l}$.

(b) Let $w, x$ be as in the statement of the lemma. Then $\left|\zeta_{l}(w)\right|_{l}=|\zeta(x)|$, which implies that column sum of $C_{w}$ is same as that of $C_{x}$. 
(c) Let $w_{1}, w_{2}, x$ be as in the statement of the lemma. Then $\zeta_{l}\left(w_{1}\right)$ and $\zeta_{l}\left(w_{2}\right)$ differ only when the length $l$ words starting at some position in $\zeta(x)$ are not subwords of $\zeta(x)$. If $|\zeta(x)| \geq l$, then the first time such a word occurs is when it starts at position $(l-1)$ from the end of $\zeta(x)$. If $|\zeta(x)|<l$, then $\zeta_{l}\left(w_{1}\right)$ and $\zeta_{l}\left(w_{2}\right)$ can differ in at most $|\zeta(x)|<l$ length $l$ words. Thus there are at most $(l-1)$ such words. Replace $\zeta, \zeta_{l}$ by $\zeta^{n},\left(\zeta^{n}\right)_{l}$ above to conclude that entries of $C_{n, w_{1}}$ and $C_{n, w_{2}}$ also differ at most by $(l-1)$.

Lemma 8.7. If $Q$ is a $s \times s$ matrix such that absolute values of all its entries are bounded above by $\delta>0$, then the absolute values of the eigenvalues of $Q$ are bounded above by $s \delta$.

Proof. Let $\lambda \neq 0$ be an eigenvalue of $Q$ and let $v=\left(v_{1}, \ldots, v_{s}\right)$ be a corresponding eigenvector. Let $r_{i}$ denote rows of $Q$. Then $\left|r_{i} \cdot v\right|=\left|\lambda v_{i}\right|$ which gives $\left|\lambda v_{i}\right| \leq$ $\delta \sum_{j=1}^{s}\left|v_{j}\right|$ for every $1 \leq i \leq s$. Adding all the inequalities together we get $|\lambda| \leq$ $s \delta$.

For every $B_{i} \subset \mathbb{A}$, let $\widetilde{B_{i}} \subset \mathbb{A}_{l}$ be the set of all words $w$ that start with a letter in $B_{i}$ and such that $w$ does not cross $B_{j}$ for any $j<i$. For every $B_{i} \subset \mathbb{A}$, let $\overline{B_{i}} \subset \mathbb{A}_{l}$ be the set of all words $w$ that start with a letter in $B_{i}$ and there exists a $j<i$ such that $w$ crosses $B_{j}$ (note that $\overline{B_{0}}$ is empty). Then we have that $\widetilde{B_{i}} \cup \overline{B_{i}}$ is the union of all words of length $l$ that start with a letter in $B_{i}$. The partial order on $\mathbb{A}_{l}$ defined earlier gives that $\widetilde{B_{0}}>\overline{B_{1}}>\widetilde{B_{1}}>\cdots>\overline{B_{k}}>\widetilde{B_{k}}$. The matrix $M_{l}$ is lower triangular block diagonal with respect to this partial order on $\mathbb{A}_{l}$. For a subset $S \subset \mathbb{A}_{l}$, denote by $S$ the transition matrix of $\zeta_{l}$ restricted to $S$.

\section{Lemma 8.8.}

(a) For every $0 \leq i \leq k$, the characteristic polynomial of $B_{i}$ divides the characteristic polynomial of $\widetilde{B_{i}}$.

(b) The eigenvalues of $\widetilde{B_{i}}$ are those of $B_{i}$ with possibly some additional eigenvalues of absolute value less than or equal to one.

(c) The eigenvalues of $\overline{B_{i}}$ have absolute value less than or equal to one.

Proof.

(a) Consider the matrix $P_{i}=\widetilde{B_{i}}-\lambda I$. We will do certain row and column operations on this matrix to reduce it to a lower triangular block diagonal matrix with $B_{i}-\lambda I$ as a diagonal block, which would imply that the characteristic polynomial of $B_{i}$ divides the characteristic polynomial of $\widetilde{B_{i}}$. For later use we denote the other diagonal block of $P_{i}$ by $Q$.

We first perform the following row operations: for every $x \in B_{i}$, choose a word $w \in \widetilde{B_{i}}$ such that $w$ starts with $x$. For every such $w$, replace the row $R_{w}$ of $\widetilde{B_{i}}$ by the sum of rows $R_{u}$ for all $u \in \widetilde{B_{i}}$ that start with $x$. Rearrange the rows and columns such that top left block is indexed by the chosen words $w$. The rearranged matrix is denoted by $P_{i}^{\prime}$. The top left block of $P_{i}^{\prime}$ is exactly $B_{i}-\lambda I$. Indeed, suppose $w, u \in \widetilde{B_{i}}$ in the top left block of $P_{i}^{\prime}$ start with $x, y \in B_{i}$, respectively. Then $P_{i}^{\prime}(w, v)$ is exactly the number of occurrences of $x$ in $\zeta(y)$.

Now for any two columns $C_{w_{1}}$ and $C_{w_{2}}$ of $P_{i}^{\prime}$, where $w_{1}, w_{2}$ start with the same letter in $B_{i}$, the first few entries (as many as the number of rows in the top left block of $P_{i}^{\prime}$ ) are equal. Now perform column operations as 
follows: for every $x \in B_{i}$ and the chosen word $w$ in the top left block, subtract $C_{w}$ from $C_{u}$ for every $u \neq w$ that start with $x$. Thus we have a lower triangular block diagonal matrix, again denoted $P_{i}^{\prime}$, with diagonal blocks $B_{i}-\lambda I$ and $Q$.

(b) Consider the lower block diagonal matrix $P_{i}^{\prime}$ from above. Eigenvalues of $P_{i}^{\prime}$ not coming from the block $B_{i}-\lambda I$ come from the lower block, denoted $Q$. By Lemma 8.6(c), the entries of $Q$ are bounded in absolute value by $(l-1)$. We claim that the eigenvalues of $Q$ are bounded in absolute value by one.

Let $\lambda_{0}$ be an eigenvalue of $Q$ and hence of $\widetilde{B_{i}}$. Then for $n \geq 1, \lambda_{0}^{n}$ is an eigenvalue of $\left(\widetilde{B_{i}}\right)^{n}$ which is a diagonal block of $\left(M_{l}\right)^{n}=\left(M^{n}\right)_{l}$. Thus $\lambda_{0}^{n}$ is an eigenvalue of $\left(\widetilde{B_{i}}\right)^{n}$ that does not come from eigenvalue of $B_{i}^{n}$, the corresponding diagonal block of $M^{n}$. Applying part $(a)$ to $\zeta^{n},\left(\widetilde{B_{i}}\right)^{n}$ can also be put in a lower triangular block diagonal form with diagonal blocks $B_{i}^{n}-\lambda I$ and $Q^{\prime}$. Since the entries of $Q^{\prime}$ are bounded by $(l-1)$, by Lemma 8.7. every eigenvalue of $Q^{\prime}$ is bounded in absolute value by size of $Q^{\prime}$ times $(l-1)$. Thus $\left|\lambda_{0}^{n}\right|$ is uniformly bounded which can happen only when $\left|\lambda_{0}\right| \leq 1$.

Thus all eigenvalues of $\widetilde{B_{i}}$ are eigenvalues of $B_{i}$ with the exception of some eigenvalues whose absolute value is less than or equal to one.

(c) Let $\lambda$ be an eigenvalue of $\overline{B_{i}}$. Then $\lambda^{n}$ is an eigenvalue of $\left(\overline{B_{i}}\right)^{n}$, the diagonal block of $\left(M^{n}\right)_{l}$ corresponding to words that start with a letter in $B_{i}$ and there exists a $j<i$ such that they cross $B_{j}$. For every $n$, the entries of $\left(\overline{B_{i}}\right)^{n}$ are bounded by $(l-1)$. Indeed, if $w$ is a length $l$ word that starts with $x$, then only the words that start at some position less than $l$ away from the last letter of $\zeta^{n}(x)$ belong to $\left(\overline{B_{i}}\right)^{n}$. This implies that eigenvalues of $\left(\overline{B_{i}}\right)^{n}$ are uniformly bounded. That is, $\left|\lambda^{n}\right|$ is uniformly bounded which can happen only when $|\lambda| \leq 1$.

Proof of Proposition 8.5. Since eigenvalues of a lower triangular block diagonal matrix are obtained from eigenvalues of each block the proposition follows from Lemma 8.8 .

8.2.2. Frequency of words. The main result in this subsection is Proposition 8.12 , Recall that we want to understand the frequency of occurrence of words which cross $B_{0}$ in $\rho$. Let $\lambda$ be the top eigenvalue of the block $B_{0}$ of $M$. Consider a subset $\mathcal{B}_{l}:=\widetilde{B_{0}} \cup\left(\bigcup_{i=1}^{k} \overline{B_{i}}\right)$ of $\mathbb{A}_{l}$. Then the set of all length $l$ words that cross $B_{0}$ is a subset of $\mathcal{B}_{l}$. The transition matrix of $\zeta_{l}$ restricted to $\mathcal{B}_{l}$ is also lower triangular block diagonal with respect to the order $\widetilde{B_{0}}>\overline{B_{1}}>\ldots>\overline{B_{k}}$ of words in $\mathcal{B}_{l}$. Then by Lemma 8.8, $\lambda>1$ is the top eigenvalue of $\mathcal{B}_{l}$ with multiplicity one. Since $\mathcal{B}_{l}$ is a diagonal block of $M_{l}$, we have $M_{l}^{n}(w, \alpha)=\mathcal{B}_{l}^{n}(w, \alpha)$ for all $w, \alpha \in \mathbb{A}_{l}$ that cross $B_{0}$.

For $w, v$ words on $\mathbb{A}$ or $\mathbb{A}_{l}$ let $(w, v)$ denote the number of occurrences of $w$ in $v$.

Lemma 8.9. Let $a \in B_{0}$ and let $\rho_{a}=\lim _{n \rightarrow \infty} \zeta^{n}(a)$ be such that $\zeta\left(\rho_{a}\right)=\rho_{a}$. Let $w \in \mathbb{A}_{l}$ be a word that crosses $B_{0}$. Then

$$
\text { frequency of occurrence of } w \text { in } \rho_{a}=\lim _{n \rightarrow \infty} \frac{\left(w, \zeta^{n}(a)\right)}{\lambda^{n}}=: d_{w, a}
$$

exists and is non-negative. Here $\lambda$ is the top eigenvalue of $B_{0}$. 
Proof. Let $\alpha \in \mathbb{A}_{l}$ start with $a$. For $n$ large, the number of occurrences of $w$ in $\zeta^{n}(a)$ is approximately the same as the number of occurrences of $w$ in $\zeta_{l}^{n}(\alpha)$. Also,

$$
\left(w, \zeta_{l}^{n}(\alpha)\right)=M_{l}^{n}(w, \alpha)
$$

We have

$$
\lim _{n \rightarrow \infty} \frac{\left(w, \zeta^{n}(a)\right)}{\lambda^{n}}=\lim _{n \rightarrow \infty} \frac{\left(w, \zeta_{l}^{n}(\alpha)\right)}{\lambda^{n}}=\lim _{n \rightarrow \infty} \frac{M_{l}^{n}(w, \alpha)}{\lambda^{n}}=\lim _{n \rightarrow \infty} \frac{\mathcal{B}_{l}^{n}(w, \alpha)}{\lambda^{n}}=: d_{w, a} .
$$

Indeed, the limit exists because $\lambda$ is the top eigenvalue of $\mathcal{B}_{l}$. The limit is nonnegative because it is a sequence of non-negative numbers. The limit does not depend on the exact choice of $\alpha$ because by Lemma 8.6(c), any two columns of $M_{l}^{n}$ starting with the same letter in $\mathbb{A}$ differ by a bounded amount and thus give the same limit.

Lemma 8.10 (Kirchhoff's Law). Let $a \in B_{0}$. Let $w \in \mathbb{A}_{l}$ cross $B_{0}$. Let we and ew be length one extensions of $w$ by $e \in \mathbb{A}$. Then

$$
d_{w, a}=\sum_{e \in \mathbb{A}} d_{w e, a}=\sum_{e \in \mathbb{A}} d_{e w, a} .
$$

Proof. We have $\left(w, \zeta^{n}(a)\right)$ and $\sum_{e \in \mathbb{A}}\left(w e, \zeta^{n}(a)\right)$ differ only when $\zeta^{n}(a)$ ends in $w$, so their difference is at most one. Thus,

$$
\left|\frac{\left(w, \zeta^{n}(a)\right)}{\lambda^{n}}-\sum_{e \in \mathbb{A}} \frac{\left(w e, \zeta^{n}(a)\right)}{\lambda^{n}}\right| \rightarrow 0 \text { as } n \rightarrow \infty,
$$

which implies that $d_{w, a}=\sum_{e \in \mathbb{A}} d_{w e, a}$. Similarly, $d_{w, a}=\sum_{e \in \mathbb{A}} d_{e w, a}$.

Lemma 8.11. Let $a, b \in B_{0}$ be distinct. Then

$$
d_{w, b}=\kappa d_{w, a}
$$

for every word $w$ that crosses $B_{0}$, where $\kappa=\kappa\left(a, b,\left.\zeta\right|_{B_{0}}\right)$.

Proof. Let us first consider the case when length of $w$ is one. We have $\zeta$ restricted to $B_{0}$ is primitive with top eigenvalue $\lambda>1$. Then

$$
d_{w, a}=\lim _{n \rightarrow \infty} \frac{M^{n}(w, a)}{\lambda^{n}}=\lim _{n \rightarrow \infty} \frac{B_{0}^{n}(w, a)}{\lambda^{n}} .
$$

Since $B_{0}$ is primitive, the limit of $B_{0}^{n} / \lambda^{n}$ is a matrix $P$ that is spanned by a positive eigenvector corresponding to $\lambda$. Since left eigenvector of $B_{0}$ is also positive, all columns of $P$ are positive multiples of each other. Thus $d_{w, b}=P(w, b)$ is a scalar multiple of $d_{w, a}=P(w, a)$ which does not depend on $w$. We call this constant $\kappa_{1}$.

Now consider the case when length of $w$ is $l$. We will first show that the constant $\kappa_{l}$, where $d_{w, b}=\kappa_{l} d_{w, a}$, does not depend on $w$ and then we will show that $\kappa_{l}=\kappa_{1}$ for all $l \geq 2$. Since $\lambda$ is the unique top eigenvalue of $\mathcal{B}_{l}, \lim _{n \rightarrow \infty} \mathcal{B}_{l}^{n} / \lambda^{n}$ is a matrix $P$ whose column span is an eigenvector corresponding to $\lambda$. Thus $d_{w, b}=P(w, b)$ is a scalar multiple of $d_{w, a}=P(w, a)$ which does not depend on $w$. We call this constant $\kappa_{l}$.

Now we will show that $\kappa_{l}=\kappa_{1}$. Let $w$ be a word of length one. We have $d_{w, b}=\sum_{e \in \mathbb{A}} d_{w e, b}$. Also $d_{w, b}=\kappa_{1} d_{w, a}$ and $d_{w e, b}=\kappa_{2} d_{w e, a}$. Thus we have $\kappa_{1} d_{w, a}=\kappa_{2} \sum_{e \in \mathbb{A}} d_{w e, a}=\kappa_{2} d_{w, a}$ which implies $\kappa_{2}=\kappa_{1}$. We can repeat the same argument to get $\kappa_{l}=\kappa_{1}$ for every $l \geq 2$.

To summarize the results about substitutions we have the following proposition. 
Proposition 8.12. Let $\zeta$ be a substitution on an alphabet $\mathbb{A}$ such that the transition matrix is lower triangular block diagonal with top left block $B_{0}$ primitive, and for every $e \in B_{0}, \zeta(e)$ starts and ends with a letter in $B_{0}$. Then there is a fixed infinite word $\rho$ obtained by iterating a letter in $B_{0}$ under $\zeta$. Moreover, the frequency of a word $w$ on $\mathbb{A}$ in $\rho$ that crosses $B_{0}$ is well defined up to scale and satisfies Kirchhoff's law.

8.2.3. Train track map as a substitution. Let $\Phi$ be a free group outer automorphism. Let $\phi: G \rightarrow G$ be a completely split train track representative of $\Phi$ with filtration $\emptyset=G_{0} \subset G_{1} \subset \cdots \subset G_{K}=G$. The transition matrix for $\phi$, denoted $M_{\phi}$, is lower triangular block diagonal. Let $a$ be an edge in an EG stratum $H_{r}$ such that up to taking powers $\phi(a)$ starts with $a$. Let $\rho_{a}=\lim _{n \rightarrow \infty} \phi^{n}(a)$. We want to understand the frequency of occurrence of paths in $G_{r}$ that cross $H_{r}$ and appear in $\rho_{a}$. We may not be able to treat $\phi$ as a substitution directly since there could be cancellations and inverse of edges would have to be treated separately. The proof of the next proposition explains how to view a completely split train track map as a substitution for the purpose of calculating frequencies of certain paths.

We set up some notation about exceptional paths that will be used in the next proposition. Let $e_{1}, e_{2} \in G$ be two linear edges such that $\phi\left(e_{1}\right)=e_{1} \sigma^{d_{1}}$ and $\phi\left(e_{2}\right)=e_{2} \sigma^{d_{2}}$, where $\sigma$ is an INP and $d_{1} \neq d_{2}$. If $d_{1}, d_{2}>0$, then $x_{m}=e_{1} \sigma^{m} \bar{e}_{2}$ where $m \in \mathbb{Z}$ is an exceptional path. We say $x_{m}$ has width $|m|$. Let $\delta=d_{1}-d_{2}$. Then $\phi\left(x_{m}\right)$ is the exceptional path $x_{m+\delta}$.

Proposition 8.13. Let $\phi: G \rightarrow G$ be a completely split train track map. Let a be an edge in an $E G$ stratum $H_{r}$ such that $\phi(a)$ starts with $a$, and let $\rho_{a}:=\lim _{n \rightarrow \infty} \phi^{n}(a)$. Let $\gamma$ be a path in $G_{r}$ that crosses $H_{r}$. Then

$$
\lim _{n \rightarrow \infty} \frac{\left(\gamma, \phi^{n}(a)\right)}{\lambda^{n}}=: d_{\gamma, a}
$$

exists and is non-negative. Here $\lambda$ is the Perron-Frobenius eigenvalue of the aperiodic EG stratum $H_{r}$. If $b \in H_{r}$ is another edge, then for every $\gamma$ as above,

$$
d_{\gamma, b}=\kappa d_{\gamma, a}
$$

where $\kappa$ is a constant with $\kappa=\kappa\left(a, b,\left.\phi\right|_{H_{r}}\right)$.

Proof. We will first show how to obtain a substitution from the completely split train track map $\phi$. Then applying Proposition 8.12 to this substitution concludes the proof. The ray $\rho_{a}$ is completely split and the terms of the complete splitting, called splitting units, of $\rho_{a}$ form an alphabet $\mathbb{A}_{\infty}$ for a substitution. But $\mathbb{A}_{\infty}$ can be infinite if there are exceptional paths. We will define a finite alphabet $\mathbb{A}_{\gamma}$, which depends on $\gamma$, by identifying some elements in $\mathbb{A}_{\infty}$ in order to calculate the frequency of occurrence of $\gamma$ in $\rho_{a}$. We will also show that the frequency of $\gamma$ in $\rho_{a}$ does not depend on the choice of the alphabet $\mathbb{A}_{\gamma}$. Let $\mathcal{N}$ be the set of all INPs, $r$-taken connecting paths and exceptional paths that appear in $\rho_{a}$.

Before we define the alphabet $\mathbb{A}_{\gamma}$, we define a relation from the set of all finite paths in $\rho_{a}$ that cross $H_{r}$, denoted $\mathcal{P}_{r}\left(\rho_{a}\right)$, to the set of all finite words on $\mathbb{A}_{\infty}$, denoted $\mathcal{W}\left(\mathcal{A}_{\infty}\right)$,

$$
r: \mathcal{P}_{r}\left(\rho_{a}\right) \rightarrow \mathcal{W}\left(\mathcal{A}_{\infty}\right)
$$

For a finite path $\gamma \in \mathcal{P}_{r}\left(\rho_{a}\right)$, the set $r(\gamma)$ consists of the following words:

(a) If an occurrence of $\gamma$ in $\rho_{a}$ is a concatenation of splitting units, then $r(\gamma)$ contains the corresponding word on $\mathbb{A}_{\infty}$. 
(b) If an occurrence of $\gamma$ in $\rho_{a}$ is a subword of an INP $\sigma$, then $r(\gamma)$ contains the element of $\mathbb{A}_{\infty}$ determined by $\sigma$, denoted $w_{\sigma}$. There are only finitely many INPs that appear in $\rho_{a}$ therefore the number of occurrences of a path $\gamma$ in an INP is bounded. If $\sigma$ contains $n$ occurrences of $\gamma$, then we let $r(\gamma)$ contain $n$ copies of $w_{\sigma}$. Note that a path $\gamma$ in $\mathcal{P}_{r}\left(\rho_{a}\right)$ is not contained in an exceptional path or an $r$-taken connected path.

(c) If an occurrence of $\gamma$ has partial overlaps with some elements of $\mathcal{N}$, then consider a path $\gamma^{\prime}$ such that $\gamma^{\prime}$ is the smallest subpath of $\rho_{a}$ that is a concatenation of splitting units and which contains $\gamma$. Then $r(\gamma)$ contains the word on $\mathbb{A}_{\infty}$ corresponding to $\gamma^{\prime}$.

Thus every occurrence of $\gamma$ in $\rho_{a}$ corresponds to the occurrence of some word from $r(\gamma)$ in $\rho_{a}$. Note that $r(\gamma)$ can be an infinite set, for instance, when $\gamma$ has partial overlap with infinitely many exceptional paths in $\rho_{a}$. But the set of words in $r(\gamma)$ viewed in the alphabet $\mathbb{A}_{\gamma}$, defined below, will form a finite set. We now define the alphabet $\mathbb{A}_{\gamma}$. For simplicity, let us assume that $\gamma$ intersects only one family of exceptional paths, say determined by linear edges $e_{1}, e_{2} \in G$.

- Let $\mathcal{H}=\left\{H_{r}=H_{i_{1}}, \ldots, H_{i_{k}}\right\}$ be the collection of strata crossed by edges in $H_{r}$. For every $H_{i_{j}}$, let $\mathbb{A}\left(H_{i_{j}}\right)$ be the alphabet which contains an edge and its inverse as distinct letters if they both appear in $\rho_{a}$ otherwise the edge with the orientation that appears.

An edge in $G$ is called a Type 1 edge if it always appears with positive or negative orientation but not both in $\rho_{a}$. An edge which appears with both orientations in $\rho_{a}$ is said to be of Type 2. If $H_{i_{j}}$ is an EG stratum, then either all edges in $H_{i_{j}}$ are Type 1 or all are Type 2 (see Uya14 for proof). Thus a substitution on $\mathbb{A}\left(H_{r}\right)$ representing $\phi$ restricted to $H_{r}$ is primitive.

- Now consider splitting units which are INPs, $r$-taken connecting paths and exceptional paths. Let $\mathbb{A}\left(\mathcal{N}_{\gamma}\right)$ be an alphabet defined as follows:

(a) All oriented INPs and $r$-taken connecting paths that appear in $\rho_{a}$ are contained $\mathbb{A}\left(\mathcal{N}_{\gamma}\right)$. There can be infinitely many INPs in $G_{r}$ but only finitely many appear in $\rho_{a}$.

(b) Suppose $\gamma$ contains an exceptional path determined by $e_{1}, e_{2}$ or a subsegment of an exceptional path determined by $e_{1}, e_{2}$. Let $N$ be the maximum length of such an exceptional path that appears in $\gamma$, in $\phi(e)$ for all edges $e$ in $H_{r}$ and in an $r$-taken connecting path. Then $\mathbb{A}\left(\mathcal{N}_{\gamma}\right)$ contains exceptional paths determined by $e_{1}, e_{2}$ of width less than or equal to $N+1$ as distinct elements. All other exceptional paths determined by $e_{1}, e_{2}$ of width greater than $N+1$ correspond to a single element of $\mathbb{A}\left(\mathcal{N}_{\gamma}\right)$.

(c) Suppose $\gamma$ does not intersect an exceptional path determined by $e_{1}, e_{2}$. Then all exceptional paths determined by $e_{1}, e_{2}$ correspond to a single element of $\mathbb{A}\left(\mathcal{N}_{\gamma}\right)$.

- Let $\mathbb{A}_{\gamma}$ be defined as the set $\mathbb{A}\left(H_{i_{1}}\right) \sqcup \cdots \sqcup \mathbb{A}\left(H_{i_{k}}\right) \sqcup \mathbb{A}\left(\mathcal{N}_{\gamma}\right)$ and let $\zeta_{\gamma, \phi}$ be a substitution on $\mathbb{A}_{\gamma}$ determined by $\phi$. Let $\tilde{r}(\gamma)$ be the set of words in $r(\gamma)$ viewed in the alphabet $\mathbb{A}_{\gamma}$. Then $\tilde{r}(\gamma)$ is a finite set of words on $\mathbb{A}_{\gamma}$. The frequency of occurrence of a path $\gamma \in \mathcal{P}_{r}\left(\rho_{a}\right)$ in $\rho_{a}$ is given by the sum of the frequencies of the words in $\tilde{r}(\gamma)$.

If we replace $N+1$ by $N+C$ for any $C \geq 1$ in the above construction to get a different alphabet $\mathbb{A}_{\gamma}^{\prime}$, then the frequency of $\gamma$ calculated from the two alphabets 
is the same. More precisely, let $\mathbb{A}_{\gamma}$ and $\mathbb{A}_{\gamma}^{\prime}$ be two alphabets which differ only in the naming of exceptional paths determined by $e_{1}, e_{2}$ of length greater than $N+1$. Let $\zeta$ and $\zeta^{\prime}$ be the corresponding substitutions, and let $\tilde{r}(\gamma)$ and $\tilde{r}^{\prime}(\gamma)$ be the set of words in $r(\gamma)$ viewed in $\mathbb{A}_{\gamma}$ and $\mathbb{A}_{\gamma}^{\prime}$, respectively. An exceptional path maps to another exceptional path under $\phi$. Therefore $\zeta$ and $\zeta^{\prime}$ have the same growth rate when restricted to $\mathbb{A}\left(H_{r}\right)$. Since the number of occurrences of $\gamma$ does not change, we get that the two substitutions yield the same frequency for words in $\tilde{r}(\gamma)$ and $\tilde{r}^{\prime}(\gamma)$ and hence the same frequency for $\gamma$.

Thus, we have obtained an alphabet $\mathbb{A}_{\gamma}$. The completely split train track map $\phi$ induces a substitution $\zeta_{\gamma}$ on this alphabet. Now Proposition 8.12 can be applied to $\zeta_{\gamma}$ to compute the frequency of occurrence of $\gamma$ in $\rho_{a}$. Different substitutions constructed here for different words $\gamma$ differ only in exceptional paths. Since an exceptional path maps to another exceptional path these different substitutions have the same growth rate when restricted to $\mathbb{A}\left(H_{r}\right)$. Also, Kirchhoff's law still holds for frequencies of paths in $\rho_{a}$ because $\left(\gamma, \phi^{n}(a)\right)$ and $\sum_{e \in G_{r}}\left(\gamma e, \phi^{n}(a)\right)$ differ by a bounded amount.

We give some examples below to exhibit how to view a completely split train track map as a substitution.

Example 8.14. Let $R_{3}$ be the rose on three petals with labels $a, b, c$. Consider a homotopy equivalence $\phi: R_{3} \rightarrow R_{3}$ given by

$$
\phi(a)=a, \phi(b)=B a c, \phi(c)=C B a c .
$$

Here capital letters denote inverses. The transition matrix for $\phi$ is

$$
\left.\begin{array}{lll}
b & c & a \\
1 & 2 & 0 \\
1 & 1 & 0 \\
1 & 1 & 1
\end{array}\right] .
$$

There are two strata $H_{1}=\{a\}$ and $H_{2}=\{b, c\}$. Every edge in $H_{2}$ is of Type 2. Let $\rho_{C}=\lim _{n \rightarrow \infty} \phi^{n}(C)$. We have $\mathcal{H}=\left\{H_{2}, H_{1}\right\}, \mathbb{A}\left(H_{2}\right)=\{b, c, B, C\}$ and $\mathbb{A}\left(H_{1}\right)=\{a, A\}$. Since there are no exceptional paths, we use one alphabet $\mathbb{A}=$ $\{b, c, B, C, a, A\}$ and a substitution $\zeta_{\phi}$ on $\mathbb{A}$ whose transition matrix is given by

$$
\left[\begin{array}{cccccc}
b & c & B & C & a & A \\
0 & 0 & 1 & 1 & 0 & 0 \\
1 & 1 & 0 & 1 & 0 & 0 \\
1 & 1 & 0 & 0 & 0 & 0 \\
0 & 1 & 1 & 1 & 0 & 0 \\
1 & 1 & 0 & 0 & 1 & 0 \\
0 & 0 & 1 & 1 & 0 & 1
\end{array}\right]
$$

Example 8.15. Consider a homotopy equivalence $\phi: R_{5} \rightarrow R_{5}$ given by

$$
\phi(a)=a b, \phi(b)=b a b, \phi(c)=c a e, \phi(d)=d c \sigma d, \phi(e)=d c a e,
$$

where $\sigma=a b A B$ is a Nielsen path. There are two strata $H_{1}=\{a, b\}$ and $H_{2}=\{c, d, e\}$. Let $\rho_{c}=\lim _{n \rightarrow \infty} \phi^{n}(c)$. We have $\mathcal{H}=\left\{H_{2}, H_{1}\right\}, \mathbb{A}\left(H_{2}\right)=$ $\{c, d, e\}, \mathbb{A}\left(H_{1}\right)=\{a, b\}$ and $\mathbb{A}(\mathcal{N})=\{\sigma\}$. Since there are no exceptional paths, we use one alphabet $\mathbb{A}=\{c, d, e, a, b, \sigma\}$ and a substitution $\zeta_{\phi}$ on $\mathbb{A}$ whose transition 
matrix is given by

$$
\left[\begin{array}{llllll}
c & d & e & a & b & \sigma \\
1 & 1 & 1 & 0 & 0 & 0 \\
0 & 2 & 1 & 0 & 0 & 0 \\
1 & 0 & 1 & 0 & 0 & 0 \\
1 & 0 & 1 & 1 & 1 & 0 \\
0 & 0 & 0 & 1 & 2 & 0 \\
0 & 1 & 0 & 0 & 0 & 1
\end{array}\right] .
$$

In this example, the frequency of occurrence of the edge path $c a$ in $\rho_{c}$ comes from the occurrence of the words $c a$ and $c \sigma$ in $\rho_{c}\left(\zeta_{\phi}\right)$. Thus the frequency of $c a$ in $\rho_{c}$ is equal to $d_{c a, c}+d_{c \sigma, c}$.

Example 8.16. This example illustrates the discussion of exceptional paths in Proposition 8.13. Consider a homotopy equivalence $\phi: R_{6} \rightarrow R_{6}$ given by

$$
\begin{array}{ll}
\phi(a)=a b, & \phi(b)=b a b, \\
\phi(c)=c \sigma^{2}, & \phi(d)=d \sigma \\
\phi(e)=e a f, & \phi(f)=f c \sigma \text { Dea } f,
\end{array}
$$

where $\sigma=a b A B$. Some exceptional paths are $x_{i}=c \sigma^{i} D$ for $i>0$. To calculate the frequency of words like $f x_{4}$ or $f c \sigma^{4}$ in $\rho_{f}$, consider the alphabet $\mathbb{A}=$ $\left\{e, f, a, b, c, D, x_{1}, x_{2}, x_{3}, x_{4}, x_{5}, \sigma, \bar{\sigma}\right\}$ and substitution $\zeta$ such that

$$
\begin{array}{ll}
\zeta(a)=a b, & \zeta(b)=b a b, \\
\zeta(c)=c \sigma^{2}, & \zeta(d)=d \sigma, \\
\zeta(f)=f x_{1} e a f, & \zeta(e)=e a f, \\
\zeta(\sigma)=\sigma, & \zeta(\bar{\sigma})=\bar{\sigma}, \\
\zeta\left(x_{i}\right)=x_{i+1} & \text { for } 1 \leq i \leq 3 . \\
\zeta\left(x_{4}\right)=\zeta\left(x_{5}\right)=x_{5}, &
\end{array}
$$

The path $\gamma=f c \sigma^{4}$ does not occur as a concatenation of splitting units in $\rho_{f}$. The path $\gamma^{\prime}=f x_{4}$ is the smallest subpath of $\rho_{f}$ that is a concatenation of splitting units and contains $\gamma$. Thus the frequency of occurrence of $\gamma$ is the same as the frequency of occurrence of $\gamma^{\prime}$.

8.3. Example of an outer automorphism relative to $\mathcal{A}$ when $\operatorname{rank}(\mathcal{A})=$ $\operatorname{rank}(\mathbb{F})$. This is an example of a relative fully irreducible outer automorphism when rank of cofactor of $\mathcal{A}$ is zero. Let $\mathbb{F}=\langle a, b, c\rangle$ and let $\mathcal{A}=\{[\langle a\rangle],[\langle b\rangle],[\langle c\rangle]\}$. Let $\Phi$ be an outer automorphism given by

$$
\Phi(a)=a, \Phi(b)=a C b c A, \Phi(c)=C b c B c .
$$

Let $\phi: G \rightarrow G$ be a relative train track representative of $\Phi$ with $G$ as in Figure 2 ,
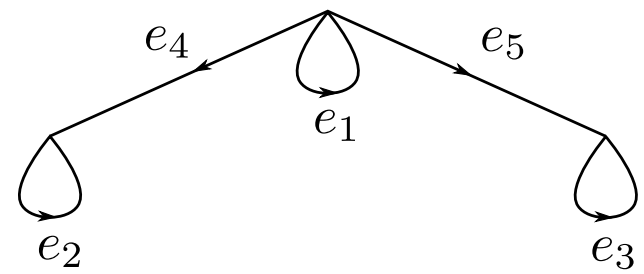

Figure 2. The graph $G$ 
The marking is given by

$$
a \rightarrow e_{1}, b \rightarrow e_{1} e_{4} e_{2} E_{4} E_{1}, c \rightarrow e_{5} e_{3} E_{5} .
$$

The map $\phi$ is given by

$$
\begin{array}{lll}
\phi\left(e_{1}\right)=e_{1}, & \phi\left(e_{2}\right)=e_{2}, & \phi\left(e_{3}\right)=e_{3}, \\
\phi\left(e_{4}\right)=e_{5} E_{3} E_{5} e_{1} e_{4}, & \phi\left(e_{5}\right)=e_{5} E_{3} E_{5} e_{1} e_{4} e_{2} E_{4} E_{1} e_{5}, &
\end{array}
$$

and the transition matrix for $\phi$ is

$$
\begin{aligned}
& \begin{array}{lllll}
e_{5} & e_{4} & e_{3} & e_{2} & e_{1}
\end{array} \\
& {\left[\begin{array}{lllll}
3 & 2 & 0 & 0 & 0 \\
2 & 1 & 0 & 0 & 0 \\
1 & 1 & 1 & 0 & 0 \\
1 & 0 & 0 & 1 & 0 \\
2 & 1 & 0 & 0 & 1
\end{array}\right] .}
\end{aligned}
$$

\section{ACKNOWLEDGMENTS}

I am grateful to my advisor Mladen Bestvina for his guidance, support and endless patience throughout this project. I would also like to thank Gilbert Levitt for pointing out an error in an earlier version, Thomas Goller for discussions about substitution dynamics, and Derrick Wigglesworth and Jon Chaika for helpful conversations. I gratefully acknowledge Mathematical Science Research Institute, Berkeley, California, where the final stage of the present work was completed during Fall 2016.

\section{REFERENCES}

[BF14] M. Bestvina and M. Feighn, Hyperbolicity of the complex of free factors, Adv. Math. 256 (2014), 104-155, DOI 10.1016/j.aim.2014.02.001. MR.3177291

[BFH97] M. Bestvina, M. Feighn, and M. Handel, Laminations, trees, and irreducible automorphisms of free groups, Geom. Funct. Anal. 7 (1997), no. 2, 215-244, DOI 10.1007/PL00001618. MR.1445386

[BFH00] M. Bestvina, M. Feighn, and M. Handel, The Tits alternative for $\operatorname{Out}\left(F_{n}\right)$. I. Dynamics of exponentially-growing automorphisms, Ann. of Math. (2) 151 (2000), no. 2, 517-623, DOI 10.2307/121043. MR 1765705

[BH92] M. Bestvina and M. Handel, Train tracks and automorphisms of free groups, Ann. of Math. (2) 135 (1992), no. 1, 1-51, DOI 10.2307/2946562. MR 1147956

[Bon88] F. Bonahon, The geometry of Teichmüller space via geodesic currents, Invent. Math. 92 (1988), no. 1, 139-162, DOI 10.1007/BF01393996. MR931208

[Bou65] N. Bourbaki. Éléments de mathématique. Fasc. XIII. Livre VI: Intégration. Chapitres 1, 2, 3 et 4: Inégalités de convexité, Espaces de Riesz, Mesures sur les espaces localement compacts, Prolongement d'une mesure, Espaces $L^{p}$. Deuxième édition revue et augmentée. Actualités Scientifiques et Industrielles, No. 1175. Hermann, Paris, 1965.

[Bri00] P. Brinkmann, Hyperbolic automorphisms of free groups, Geom. Funct. Anal. 10 (2000), no. 5, 1071-1089, DOI 10.1007/PL00001647. MR 1800064

[Coo87] D. Cooper, Automorphisms of free groups have finitely generated fixed point sets, J. Algebra 111 (1987), no. 2, 453-456, DOI 10.1016/0021-8693(87)90229-8. MR916179

[FH11] M. Feighn and M. Handel, The recognition theorem for Out $\left(F_{n}\right)$, Groups Geom. Dyn. 5 (2011), no. 1, 39-106, DOI 10.4171/GGD/116. MR2763779

[Gup16] R. Gupta, Loxodromic elements for the relative free factor complex, arXiv:1612.04261, December 2016.

[HM13] M. Handel and L. Mosher, Subgroup decomposition in Out $\left(F_{n}\right)$ : Introduction and Research Announcement, arXiv:1302.2681, February 2013.

[HM14] M. Handel and L. Mosher, Relative free splitting and relative free factor complexes I: Hyperbolicity, arXiv:1407.3508v1, 2014. 
[Kap05] I. Kapovich, The frequency space of a free group, Internat. J. Algebra Comput. 15 (2005), no. 5-6, 939-969, DOI 10.1142/S0218196705002700. MR2197815

[Kap06] I. Kapovich, Currents on free groups, Topological and asymptotic aspects of group theory, Contemp. Math., vol. 394, Amer. Math. Soc., Providence, RI, 2006, pp. 149-176, DOI 10.1090/conm/394/07441. MR2216713

[KL09] I. Kapovich and M. Lustig, Geometric intersection number and analogues of the curve complex for free groups, Geom. Topol. 13 (2009), no. 3, 1805-1833, DOI 10.2140/gt.2009.13.1805. MR2496058

[LU15] M. Lustig and C. Uyanik. North-South dynamics of hyperbolic free group automorphisms on the space of currents, ArXiv e-prints, September 2015.

[Mar95] R. Martin, Non-uniquely ergodic foliations of thin-type, measured currents and automorphisms of free groups, ProQuest LLC, Ann Arbor, MI, 1995. Thesis (Ph.D.)-University of California, Los Angeles. MR2693216

[MM99] H. A. Masur and Y. N. Minsky, Geometry of the complex of curves. I. Hyperbolicity, Invent. Math. 138 (1999), no. 1, 103-149, DOI 10.1007/s002220050343. MR1714338

[Que87] M. Queffélec, Substitution dynamical systems - spectral analysis, Lecture Notes in Mathematics, vol. 1294, Springer-Verlag, Berlin, 1987. MR.924156

[Sta99] J. R. Stallings, Whitehead graphs on handlebodies, Geometric group theory down under (Canberra, 1996), de Gruyter, Berlin, 1999, pp. 317-330. MR1714852

[Uya14] C. Uyanik, Dynamics of hyperbolic iwips, Conform. Geom. Dyn. 18 (2014), 192-216, DOI 10.1090/S1088-4173-2014-00270-7. MR3273532

[Uya15] C. Uyanik, Generalized north-south dynamics on the space of geodesic currents, Geom. Dedicata 177 (2015), 129-148, DOI 10.1007/s10711-014-9982-2. MR3370027

[Whi36] J. H. C. Whitehead, On Certain Sets of Elements in a Free Group, Proc. London Math. Soc. (2) S2-41 (1936), no. 1, 48-56, DOI 10.1112/plms/s2-41.1.48. MR.1575455

Department of Mathematics, University of Utah, Salt Lake City, Utah 84112

Current address: Department of Mathematics, Technion, Haifa 32000, Israel

E-mail address: radhikagup@technion.ac.il 